Annales Geophysicae (2002) 20: 847-862 (c) European Geophysical Society 2002

\title{
STRATAQ: A three-dimensional Chemical Transport Model of the stratosphere
}

\author{
B. Grassi, G. Redaelli, and G. Visconti \\ Universita degli Studi di l'Aquila, Dipartimento di Fisica, Via Vetoio, 67010 Loc. Coppito l'Aquila, Italy
}

Received: 31 May 2000 - Revised: 22 October 2001 - Accepted: 30 October 2001

\begin{abstract}
A three-dimensional (3-D) Chemical Transport Model (CTM) of the stratosphere has been developed and used for a test study of the evolution of chemical species in the arctic lower stratosphere during winter 1996/97. This particular winter has been chosen for testing the model's capabilities for its remarkable dynamical situation (very cold and strong polar vortex) along with the availability of sparse chlorine, $\mathrm{HNO}_{3}$ and $\mathrm{O}_{3}$ data, showing also very low $\mathrm{O}_{3}$ values in late March/April. Due to those unusual features, the winter 1996/97 can be considered an excellent example of the impact of both dynamics and heterogeneous reactions on the chemistry of the stratosphere. Model integration has been performed from January to March 1997 and the resulting long-lived and short-lived tracer fields compared with available measurements. The model includes a detailed gas phase chemical scheme and a parameterization of the heterogeneous reactions occurring on liquid aerosol and polar stratospheric cloud (PSC) surfaces. The transport is calculated using a semi-lagrangian flux scheme, forced by meteorological analyses. In such form, the STRATAQ CTM model is suitable for short-term integrations to study transport and chemical evolution related to "real" meteorological situations. Model simulation during the chosen winter shows intense PSC formation, with noticeable local $\mathrm{HNO}_{3}$ capture by PSCs, and the activation of vortex air leading to chlorine production and subsequent $\mathrm{O}_{3}$ destruction. The resulting model fields show generally good agreement with satellite data (MLS and TOMS), although the available observations, due to their limited number and time/space sparse nature, are not enough to effectively constraint the model. In particular, the model seems to perform well in reproducing the rapid processing of air inside the polar vortex on PSC converting reservoir species in active chlorine. In addition, it satisfactorily reproduces the morphology of the continuous $\mathrm{O}_{3}$ decline as shown by the satellite during the investigated period, with a tendency, however, to underestimate the total column val-
\end{abstract}

Correspondence to: $\mathrm{B}$. Grassi

(Barbara.grassi@aquila.infn.it) ues inside the polar vortex during late winter. As possible causes of this model/observation difference we suggest an incorrect estimation of the vertical transport and of the tropospheric contribution.

Key words. Atmospheric composition and structure (Middle atmosphere-composition and chemistry) Meterology and atmospheric dynamics (middle atmosphere dynamics)

\section{Introduction}

Numerical models are commonly used to help quantify chemical and transport processes in the atmosphere. The formulation of these models varies according the type of problems to be addressed. For example, the so-called " 0 dimensional" (0-D) or "box" models are used to simulate a great number of chemical reactions at one point in the atmosphere (e.g. Danilin et al., 1994; Kawa et al., 1992; Ramaroson et al., 1992; Redaelli, 1997). Such an approach that is particularly suited for the use along the lagrangian trajectory of one or multiple traveling air masses allows one to concentrate the computational resources into a highly detailed parameterization of chemical and microphysical reactions. Opposite to those "chemistry-intensive" models, multi-dimensional "transport-intensive" models concentrate on following the evolutions of quasi-conservative tracers with a very simple parameterization of chemistry (e.g. Cunnold et al., 1975; Shindell et al., 1998). Such models, usually three-dimensional (3-D) with a different choice of the vertical coordinate, have been successfully used to study transport processes over various time scales. In between those extremes, there are one-dimensional models that study the evolution of a single air column, but usually with a crude chemical parameterization (e.g. Chameides and Cicerone, 1978), and two-dimensional (2-D) models that study the atmosphere averaging over one of the spatial coordinates, usually the longitude (e.g. Brasseur et al., 1990; Ko et al., 1993; Visconti, 1987). This latest approach has been proved to be a good 
compromise between the two extremes and has been used to study stratospheric chemistry with good detail in both chemical and microphysical parameterizations. However, those models cannot be used either to analyze specific time chemical pictures or to study single measurements, especially when the high non-zonal properties of the circulation play an important role, as in the presence of a highly disturbed polar vortex. Until recently, the development of global 3-D models with extensive chemistry has been inhibited by a lack of computer resources. This is particularly true for those types of 3-D models where the chemistry is included directly into the dynamical code (the so-called GCMs) (Austin et al., 2000, 2001; Steil et al., 1998; Hein et al., 2001). Chemical Transport Models (CTMs) are instead 3-D models where the transport is performed "off-line", meaning that those models use meteorological parameters from an external GCM, or assimilated winds and temperature provided by meteorological agencies for the period of interest, to force the chemical calculations. Such an approach is less computationally expensive, because it avoids the internal dynamical calculations and allows one to have the complete 3-D picture of transport, still concentrating on a highly detailed parameterization of the chemistry. Moreover, this approach allows for the comparisons of model output with observations at a chosen time, because it constraints the results to a "real" specific meteorological condition. This is particularly important when trying to study a great number of sparse single measurements, concentrated in a limited time period, such as during a measurement campaign. In this case, a highly detailed chemical parameterization and a good resolution of non-zonal transport are required. This type of model has performed well in reproducing experimental data and day-to-day chemical and dynamical variability of the stratospheric and tropospheric constituents (e.g. see Rood et al., 1992; Lefevre et al., 1994; Chipperfield et al., 1993).

This paper describes a global off-line three-dimensional chemical and transport model developed at the University of L'Aquila. There are several reasons to develop such a model besides the scientific competition. One reason is the involvement of our group in a few international measurement campaigns that require the use of both CTM and lagrangian trajectory modeling (MacKenzie et al., 2000; Carli et al., 2000). Another reason is the growing opportunity to obtain global or synoptic data on atmospheric components from satellites such as ENVISAT. The more attractive development in this case deals with assimilation techniques from which both models and the completeness of the data will benefit. The general belief is that models need to be tested and this is the purpose of this paper: to show how the model may reproduce some of the available measurements of the atmospheric composition. As discussed clearly in Oreskes et al. (1994), the outcome of such a comparison may not be the basis for rejecting or accepting the models. In fact, in the Earth Sciences, the verification or validation of the models poses a serious methodological problem. On the other hand, if the models fail to reproduce the data it may be a sign that they are faulty and need improvement that may come along with future applications. In addition, a few CTMs are involved in assessment works (i.e. predictions for IPCC, etc.) and still some of them have not been subjected to such an exercise. This work has to be read with this limitation in mind, as a tool that can be improved by being devoted mainly to help analyze experimental data.

The paper is organized as follows: a description of the model structure is given in Sect. 2; the formulation used to calculate transport and the chemistry scheme are then presented. In Sect. 3, the characteristics of the performed experiments are described and the results are compared with a number of observations to assess the model performance. In the final sections the results are discussed and summarized.

\section{STRATAQ chemical transport model}

STRATAQ is a three-dimensional off-line stratospheric chemical transport model that extends from $0.6 \mathrm{~km}$ to about $52 \mathrm{~km}$ in altitude. The model uses a sigma-pressure vertical coordinate and has a vertical resolution varying from about $1-1.5 \mathrm{~km}$ until $20 \mathrm{~km}$ and up to $2.5 \mathrm{~km}$ in the higher stratosphere. The horizontal resolution is $5^{\circ}$ longitude by $2^{\circ}$ latitude. The behaviour of the chemical species is represented by the continuity and transport equation which separately includes the contribution of transport by the large-scale circulation and the tendency due to the chemistry.

\subsection{Dynamic scheme}

In the CTM chemical species are advected with a semilagrangian transport code, using a Van Leer transport scheme (Van Leer, 1974; Allen et al., 1991; Lin et al., 1996) that is a monotonic, upstream-biased differencing scheme. The Lagrangian calculation, characteristic of upstream-biased schemes, makes the method ideal for mass conservation. The major advantage of the scheme is its ability to maintain constituent correlations. In fact, a monotonic grid-point transport scheme applied to 3-D constituent transport does not generate new maxima or minima and, therefore, does not require a filling algorithm. However, the major disadvantage of the scheme is that upstream calculation can be a source of numerical diffusion. The algorithm used makes the diffusion self-limiting and scale-dependent.

The equations are discretized with height on $28 \sigma$ levels (see Table 1). The transport scheme uses pure $\sigma$ vertical coordinates defined as:

$\sigma_{(i)}=\frac{\left(p_{(i)}-p_{t o p}\right)}{\delta p}$,

where $p$ is the pressure and $\delta p=p_{s f c}-p_{\text {top }}$ being $p_{\text {top }}$, the pressure at the top of the model grid box and $p_{s f c}$ the surface pressure. Note from Table 1 that the levels are not equally spaced. Species are transported with a time step of 15 min.

Analyzed horizontal wind components, obtained from the United Kingdom Meteorological Office (UKMO), are used to calculate the transport of the stratospheric species. The 
Table 1. Sigma levels of the model

\begin{tabular}{|c|c|c|c|}
\hline & $\sigma$ & $p_{i} \mathrm{hPa}$ & $z_{i} \mathrm{~km}$ \\
\hline 1 & $1.83 \mathrm{e}-7$ & 0.6 & 51.8 \\
\hline 2 & $6.78 \mathrm{e}-4$ & 1.3 & 46.8 \\
\hline 3 & $1.50 \mathrm{e}-3$ & 2.1 & 43.4 \\
\hline 4 & $2.51 \mathrm{e}-3$ & 3.2 & 40.7 \\
\hline 5 & $4.00 \mathrm{e}-3$ & 4.6 & 37.9 \\
\hline 6 & $6.16 \mathrm{e}-3$ & 35.2 & \\
\hline 7 & $9.35 \mathrm{e}-3$ & 10.0 & 32.5 \\
\hline 8 & $1.40 \mathrm{e}-2$ & 14.7 & 29.8 \\
\hline 9 & $2.09 \mathrm{e}-2$ & 21.5 & 27.1 \\
\hline 10 & $3.10 \mathrm{e}-2$ & 31.6 & 24.3 \\
\hline 11 & $4.38 \mathrm{e}-2$ & 44.4 & 22.0 \\
\hline 12 & $5.60 \mathrm{e}-2$ & 56.6 & 20.3 \\
\hline 13 & $6.66 \mathrm{e}-2$ & 67.2 & 19.1 \\
\hline 14 & $7.66 \mathrm{e}-2$ & 77.2 & 18.1 \\
\hline 15 & $8.73 \mathrm{e}-2$ & 87.9 & 17.2 \\
\hline 16 & $1.00 \mathrm{e}-1$ & 101 & 16.2 \\
\hline 17 & 0.118 & 119 & 15.0 \\
\hline 18 & 0.139 & 140 & 13.9 \\
\hline 19 & 0.161 & 162 & 12.9 \\
\hline 20 & 0.188 & 189 & 11.8 \\
\hline 21 & 0.224 & 225 & 10.5 \\
\hline 22 & 0.276 & 276 & 9.1 \\
\hline 23 & 0.348 & 348 & 7.4 \\
\hline 24 & 0.441 & 441 & 5.8 \\
\hline 25 & 0.543 & 543 & 4.3 \\
\hline 26 & 0.649 & 649 & 3.0 \\
\hline 27 & 0.772 & 772 & 1.8 \\
\hline 28 & 0.918 & 918 & 0.6 \\
\hline
\end{tabular}

UKMO three-dimensional grid-point analyses are gridded on 18 pressure levels from $1000 \mathrm{hPa}$ to $0.316 \mathrm{hPa}$ on a $2.4^{\circ}$ latitude by $3.75^{\circ}$ longitude global grid. The daily analyses (at 12:00 UTC) are generated using a data assimilation system with data from operational meteorological observations; this system is a development of the scheme used at UKMO for operational weather forecasting, which has been extended to cover the whole stratosphere (Swinbank and O"Neill, 1994). The vertical wind component is simply obtained in the transport scheme used by integrating the hydrostatic mass continuity equation.

\subsection{Stratospheric chemistry scheme}

Variation in the chemical species and families due to transport and chemistry are treated separately in the model. Each chemical species and each family is first advected and then chemically integrated.

The photochemical package used in the CTM is based on a photochemical box model, developed by Redaelli (1997), to be used for short time integration along lagrangian trajectories (Redaelli et al., 1998). The original 0-D model has been updated with more recent values for chemical constants, introducing a "family" approach and strongly simplifying the heterogeneous calculation to be less computationally expensive.

The chemical package includes 43 chemical species, 89 homogeneous gas-phase chemical reactions, relevant to the stratosphere and troposphere, and 33 photolytic reactions. No additional tropospheric-only related detailed chemistry is included in the scheme.

A family approach has been employed and a number of short-lived species have been combined together into families. In this way it is possible to save computer time and memory. The concentrations of species included in the families are calculated assuming photochemical equilibrium conditions. $\mathrm{HO}_{x}$ species $\left(\mathrm{H}, \mathrm{OH}, \mathrm{HO}_{2}\right)$ and $\mathrm{CH}_{y} \mathrm{O}_{x}$ species $\left(\mathrm{CH}_{3}, \mathrm{CH}_{3} \mathrm{O}_{2}, \mathrm{CH}_{3} \mathrm{O}, \mathrm{CH}_{2} \mathrm{O}\right)$ are not explicitly integrated but considered in a steady state. In fact, photochemical lifetimes of these species are very short throughout the stratosphere and much shorter than dynamical time scale and so their abundance will not depend directly on transport. $\mathrm{N}_{2}$, $\mathrm{O}_{2}, \mathrm{H}_{2}$ are assumed to have fixed mixing ratios. $\mathrm{H}_{2} \mathrm{O}$ evolution is roughly parameterized in the tropospheric levels, as a function of thermodynamical parameters. No surface emissions of $\mathrm{NO}_{x}$ are considered in the model, while $\mathrm{CH}_{4}, \mathrm{~N}_{2} \mathrm{O}$ and CFCs are constrained at ground and top level according to mixing ratio values used for the initialization. Total inorganic bromine and chlorine are also prescribed in the model using fixed correlation with $\mathrm{N}_{2} \mathrm{O}$. For the experiment described in Sect. 3, a constant total chlorine loading of $3.6 \mathrm{ppbv}$ and a total bromine loading of $20 \mathrm{pptv}$ has been used, following Chipperfield (1999).

To further reduce computational cost, some long-lived species are not chemically integrated but only transported. All the chemical species contained in the model are listed in Table 2, along with a description of the chemical families.

To evaluate photoanalysis rates $J$ we use a "look-up table" calculated by a radiative one-dimensional model based on the $\delta$-Eddington approximation (Joseph et al., 1976). The model includes 26 vertical levels from the ground to about $70 \mathrm{~km}$ and takes into account 68 spectral intervals. The radiative scheme takes into account Rayleigh and Mie scattering and includes the Allen-Frederick parameterization of SchumannRunge bands (Allen and Frederick, 1982). Solar flux values and absorption cross sections for the chemical compounds are taken from DeMore et al. (1997). The resulting threedimensional look-up table for $J$ values (which has coordinates of latitude, pressure altitude, and solar zenith angle with $2^{\circ}$ step) is calculated off-line for clear-sky conditions, as a 10 day average. For the calculations, climatological zonal $\mathrm{O}_{3}$ data (Pitari et al., 1998) are used. The temperature data are taken from UKMO analyses. Such precomputed $J$ values are then interpolated for each time step at each model grid point. The chemical mechanisms and rate constants are given in Tables 3 and 4. Chemical rates are also calculated using UKMO temperature data.

The model uses the semi-implicit symmetric (SIS) method to integrate the chemical continuity equations (Ramaroson 
Table 2. Chemical species contained in the model

\begin{tabular}{|c|c|}
\hline Category & Species \\
\hline $\begin{array}{r}\text { Short lived } \\
\text { species }\end{array}$ & $\begin{array}{l}\mathrm{O}_{x}\left(=\mathrm{O}_{3}+\mathrm{O}\left({ }^{3} P\right)+\mathrm{O}\left({ }^{1} D\right)\right), \mathrm{H}_{2} \mathrm{O}_{2} \\
\mathrm{NO}_{x}\left(=\mathrm{N}+\mathrm{NO}+\mathrm{NO}_{2}\right), \mathrm{NO}_{3} \\
\mathrm{~N}_{2} \mathrm{O}_{5}, \mathrm{HNO}_{3}, \mathrm{HO}_{2} \mathrm{NO}_{2} \\
\mathrm{ClO}_{x}\left(=\mathrm{Cl}+\mathrm{ClO}+2 \mathrm{Cl}_{2} \mathrm{O}_{2}\right), \mathrm{ClONO}_{2} \\
\mathrm{HCl}, \mathrm{HOCl}, \mathrm{OClO} \\
\mathrm{BrO}_{x}(=\mathrm{Br}+\mathrm{BrO}), \mathrm{HBr} \\
\mathrm{HOBr} \mathrm{BrONO}\end{array}$ \\
\hline Steady state & $\begin{array}{l}\mathrm{H}, \mathrm{OH}, \mathrm{HO}_{2} \\
\mathrm{CH}_{3}, \mathrm{CH}_{3} \mathrm{O}_{2}, \mathrm{CH}_{3} \mathrm{O} \\
\mathrm{CH}_{2} \mathrm{O}\end{array}$ \\
\hline $\begin{array}{r}\text { Long lived } \\
\text { species }\end{array}$ & $\begin{array}{l}\mathrm{CH}_{4}, \mathrm{~N}_{2} \mathrm{O}, \mathrm{CO}, \mathrm{H}_{2} \mathrm{O} \\
\mathrm{CFCl}_{3}, \mathrm{CF}_{2} \mathrm{Cl}_{2} \\
\mathrm{CH}_{3} \mathrm{Cl}, \mathrm{CH}_{3} \mathrm{Br} \\
\mathrm{CF}_{2} \mathrm{ClBr}, \mathrm{CF}_{3} \mathrm{Br}\end{array}$ \\
\hline Fixed & $\mathrm{O}_{2}, \mathrm{~N}_{2}, \mathrm{H}_{2}$ \\
\hline
\end{tabular}

et al., 1992). Noted $Q_{i}$, the photochemical source term for specie $i$, as:

$Q_{i}=P_{i}-L_{i}$,

where $P_{i}$ and $L_{i}$ are, respectively, the production and loss terms, and the defined two vectors $\boldsymbol{y}$ and $\boldsymbol{Q}$ whose components are, respectively, the concentrations $n_{i}$ and the photochemical-source terms $Q_{i}$. In the SIS method, the chemical continuity equation for a species $i$ is developed to obtain:

$y\left(t_{m+1}\right)=y\left(t_{m}\right)+\Delta t\left(Q\left(t_{m}, y\left(t_{m}\right)\right)+J_{m} \frac{\Delta y}{2}\right)$

where: $\Delta y=y\left(t_{m+1}\right)-y\left(t_{m}\right)$, and $J_{m}$ is the Jacobian matrix:

$J_{m}=\frac{\partial Q_{i}}{\partial n_{j}}$

with $i=1,2, \ldots N$ and $j=1,2, \ldots N$ being $N$ the number of the species of the photochemical system. The SIS method has the desired properties of an integration scheme: it is accurate, stable and relatively fast compared to other methods for solving stiff systems. The time step used in the CTM for the chemistry is $15 \mathrm{~min}$. Although the chemistry scheme used is stable for longer time steps, such a short time step value is necessary to adequately resolve the diurnal cycle of species.

A treatment of heterogeneous processes, occurring on the surface of stratospheric background aerosols, NAT (Nitric Acid Tetrahydrate) and ICE particles, is included in the model. The scheme does not contain any detailed microphysics, but it does distinguish between those different basic heterogeneous surfaces. In the presence of liquid aerosols
Table 3. Photolytic reactions in the model

\begin{tabular}{|c|c|c|c|}
\hline No. & Reaction & & \\
\hline 1 & $\mathrm{NO}+\mathrm{h} v$ & $\longrightarrow$ & $\mathrm{N}+\mathrm{O}$ \\
\hline 2 & $\mathrm{O}_{2}+\mathrm{h} v$ & $\longrightarrow$ & $\mathrm{O}+\mathrm{O}$ \\
\hline 3 & $\mathrm{O}_{3}+\mathrm{h} v$ & $\longrightarrow$ & $\mathrm{O}+\mathrm{O}_{2}$ \\
\hline 4 & $\mathrm{O}_{3}+\mathrm{h} v$ & $\longrightarrow$ & $\mathrm{O}\left({ }^{1} \mathrm{D}\right)+\mathrm{O}_{2}$ \\
\hline 5 & $\mathrm{CH}_{2} \mathrm{O}+\mathrm{h} v$ & $\longrightarrow$ & $\mathrm{CHO}+\mathrm{H}$ \\
\hline 6 & $\mathrm{CH}_{2} \mathrm{O}+\mathrm{h} v$ & $\longrightarrow$ & $\mathrm{CO}+\mathrm{H}_{2}$ \\
\hline 7 & $\mathrm{H}_{2} \mathrm{O}_{2}+\mathrm{h} v$ & $\longrightarrow$ & $\mathrm{OH}+\mathrm{OH}$ \\
\hline 8 & $\mathrm{NO}_{2}+\mathrm{h} v$ & $\longrightarrow$ & $\mathrm{NO}+\mathrm{O}$ \\
\hline 9 & $\mathrm{NO}_{3}+\mathrm{h} v$ & $\longrightarrow$ & $\mathrm{NO}_{2}+\mathrm{O}$ \\
\hline 10 & $\mathrm{NO}_{3}+\mathrm{h} v$ & $\longrightarrow$ & $\mathrm{NO}+\mathrm{O}_{2}$ \\
\hline 11 & $\mathrm{~N}_{2} \mathrm{O}_{5}+\mathrm{h} v$ & $\longrightarrow$ & $\mathrm{NO}_{2}+\mathrm{NO}_{3}$ \\
\hline 12 & $\mathrm{HNO}_{3}+\mathrm{h} v$ & $\longrightarrow$ & $\mathrm{NO}_{2}+\mathrm{OH}$ \\
\hline 13 & $\mathrm{HNO}_{4}+\mathrm{h} v$ & $\longrightarrow$ & $\mathrm{NO}_{2}+\mathrm{HO}_{2}$ \\
\hline 14 & $\mathrm{ClONO}_{2}+\mathrm{h} v$ & $\longrightarrow$ & $\mathrm{Cl}+\mathrm{NO}_{3}$ \\
\hline 15 & $\mathrm{HOCl}+\mathrm{h} v$ & $\longrightarrow$ & $\mathrm{Cl}+\mathrm{OH}$ \\
\hline 16 & $\mathrm{OClO}+\mathrm{h} v$ & $\longrightarrow$ & $\mathrm{O}+\mathrm{ClO}$ \\
\hline 17 & $\mathrm{Cl}_{2} \mathrm{O}_{2}+\mathrm{h} v$ & $\longrightarrow$ & $\mathrm{Cl}+\mathrm{Cl}+\mathrm{O}_{2}$ \\
\hline 18 & $\mathrm{ClO}+\mathrm{h} v$ & $\longrightarrow$ & $\mathrm{Cl}+\mathrm{O}$ \\
\hline 19 & $\mathrm{BrO}+\mathrm{h} v$ & $\longrightarrow$ & $\mathrm{Br}+\mathrm{O}$ \\
\hline 20 & $\mathrm{BrNO}_{3}+\mathrm{h} v$ & $\longrightarrow$ & $\mathrm{Br}+\mathrm{NO}_{3}$ \\
\hline 21 & $\mathrm{HOBr}+\mathrm{h} v$ & $\longrightarrow$ & $\mathrm{Br}+\mathrm{OH}$ \\
\hline 22 & $\mathrm{~N}_{2} \mathrm{O}+\mathrm{h} v$ & $\longrightarrow$ & $\mathrm{N}_{2}+\mathrm{O}$ \\
\hline 23 & $\mathrm{CFCl}_{3}+\mathrm{h} v$ & $\longrightarrow$ & Products \\
\hline 24 & $\mathrm{CF}_{2} \mathrm{Cl}_{2}+\mathrm{h} v$ & $\longrightarrow$ & Products \\
\hline 26 & $\mathrm{CH}_{3} \mathrm{Cl}+\mathrm{h} v$ & $\longrightarrow$ & $\mathrm{CH}_{3}+\mathrm{Cl}$ \\
\hline 27 & $\mathrm{CH}_{3} \mathrm{Br}+\mathrm{h} v$ & $\longrightarrow$ & $\mathrm{CH}_{3}+\mathrm{Br}$ \\
\hline 28 & $\mathrm{CF}_{2} \mathrm{ClBr}+\mathrm{h} v$ & $\longrightarrow$ & Products \\
\hline 29 & $\mathrm{CF}_{3} \mathrm{Br}+\mathrm{h} v$ & $\longrightarrow$ & Products \\
\hline 30 & $\mathrm{H}_{2} \mathrm{O}+\mathrm{h} v$ & $\longrightarrow$ & $\mathrm{OH}+\mathrm{H}$ \\
\hline 31 & $\mathrm{HO}_{2}+\mathrm{h} v$ & $\longrightarrow$ & $\mathrm{OH}+\mathrm{O}$ \\
\hline 32 & $\mathrm{HCl}+\mathrm{h} v$ & $\longrightarrow$ & $\mathrm{H}+\mathrm{Cl}$ \\
\hline 33 & $\mathrm{Cl}_{2}+\mathrm{h} v$ & $\longrightarrow$ & $\mathrm{Cl}+\mathrm{Cl}$ \\
\hline
\end{tabular}

and PSCs, the following six heterogeneous reactions are assumed to occur on the particle surfaces:

$$
\begin{aligned}
\mathrm{N}_{2} \mathrm{O}_{5}(g)+\mathrm{H}_{2} \mathrm{O}(s) & \longrightarrow \mathrm{HNO}_{3}(s)+\mathrm{HNO}_{3}(s) \\
\mathrm{N}_{2} \mathrm{O}_{5}(g)+\mathrm{HCl}(s) & \longrightarrow \mathrm{ClNO}_{2}(g)+\mathrm{HNO}_{3}(s) \\
\mathrm{ClONO}_{2}(g)+\mathrm{H}_{2} \mathrm{O}(s) & \longrightarrow \mathrm{HOCl}(g)+\mathrm{HNO}_{3}(s) \\
\mathrm{ClONO}_{2}(g)+\mathrm{HCl}(s) & \longrightarrow \mathrm{Cl}_{2}(g)+\mathrm{HNO}_{3}(s) \\
\mathrm{HOCl}(g)+\mathrm{HCl}(s) & \longrightarrow \mathrm{Cl}_{2}(g)+\mathrm{H}_{2} \mathrm{O}(s) \\
\mathrm{BrONO}_{2}(g)+\mathrm{H}_{2} \mathrm{O}(s) & \longrightarrow \mathrm{HOBr}(g)+\mathrm{HNO}_{3}(s)
\end{aligned}
$$

where $(s)$ and $(g)$ stand for solid phase and gaseous phase, respectively. The reaction probabilities $\gamma$ for these reactions are taken from experimental data as reported in DeMore et al. (1994) and Chipperfield (1999). Temperature and relative humidity-dependent sticking coefficients are calculated from the model $\mathrm{H}_{2} \mathrm{O}, T$, and $\mathrm{HCl}$ according to Hanson and Ravishankara (1994) for reactions (R3) and (R4) and Hanson et al. (1994) for reaction (R5) aver liquid aerosols. The $\gamma$ used are summarized in Table 5. The rate of chemical reaction is then 
Table 4. Chemical reaction mechanism and kinetic rates

\begin{tabular}{|c|c|c|c|c|}
\hline No. & Reaction & & & Rate \\
\hline 1 & $\mathrm{O}+\mathrm{O}+M$ & $\longrightarrow$ & $\mathrm{O}_{2}+M$ & $4.2 \cdot 10^{-28}[M] \cdot\left(\frac{1}{T}\right)^{2}$ \\
\hline 2 & $\mathrm{O}+\mathrm{O}_{2}+M$ & $\longrightarrow$ & $\mathrm{O}_{3}+M$ & $6.0 \cdot 10^{-34}[M] \cdot\left(\frac{300}{T}\right)^{2.3}$ \\
\hline 3 & $\mathrm{O}+\mathrm{O}_{3}$ & $\longrightarrow$ & $2 \mathrm{O}_{2}$ & $8.0 \cdot 10^{-12} \cdot \exp \left(\frac{-2600}{T}\right)$ \\
\hline 4 & $\mathrm{O}\left({ }^{1} \mathrm{D}\right)+\mathrm{N}_{2}$ & $\longrightarrow$ & $\mathrm{O}+\mathrm{N}_{2}$ & $1.8 \cdot 10^{-11} \cdot \exp \left(\frac{110}{T}\right)$ \\
\hline 5 & $\mathrm{O}\left({ }^{1} \mathrm{D}\right)+\mathrm{O}_{2}$ & $\longrightarrow$ & $\mathrm{O}+\mathrm{O}_{2}$ & $3.2 \cdot 10^{-11} \cdot \exp \left(\frac{70}{T}\right)$ \\
\hline 6 & $\mathrm{O}\left({ }^{1} \mathrm{D}\right)+\mathrm{H}_{2} \mathrm{O}$ & $\longrightarrow$ & $\mathrm{OH}+\mathrm{OH}$ & $2.2 \cdot 10^{-10}$ \\
\hline 7 & $\mathrm{OH}+\mathrm{O}$ & $\longrightarrow$ & $\mathrm{H}+\mathrm{O}_{2}$ & $2.2 \cdot 10^{-11} \cdot \exp \left(\frac{120}{T}\right)$ \\
\hline 8 & $\mathrm{H}+\mathrm{O}_{2}+M$ & $\longrightarrow$ & $\mathrm{HO}_{2}+M$ & $k_{0}=5.7 \cdot 10^{-32} \cdot\left(\frac{300}{T}\right)^{1.6} \cdot k_{\infty}=7.5 \cdot 10^{-11}$ \\
\hline 9 & $\mathrm{HO}_{2}+\mathrm{O}$ & $\longrightarrow$ & $\mathrm{OH}+\mathrm{O}_{2}$ & $3.0 \cdot 10^{-11} \cdot \exp \left(\frac{200}{T}\right)$ \\
\hline 10 & $\mathrm{OH}+\mathrm{O}_{3}$ & $\longrightarrow$ & $\mathrm{HO}_{2}+\mathrm{O}_{2}$ & $1.6 \cdot 10^{-12} \cdot \exp \left(\frac{-940}{T}\right)$ \\
\hline 11 & $\mathrm{H}+\mathrm{O}_{3}$ & $\longrightarrow$ & $\mathrm{OH}+\mathrm{O}_{2}$ & $1.4 \cdot 10^{-10} \cdot \exp \left(\frac{-470}{T}\right)$ \\
\hline 12 & $\mathrm{OH}+\mathrm{HO}_{2}$ & $\longrightarrow$ & $\mathrm{H}_{2} \mathrm{O}+\mathrm{O}_{2}$ & $4.8 \cdot 10^{-11} \cdot \exp \left(\frac{250}{T}\right)$ \\
\hline 13 & $\mathrm{OH}+\mathrm{OH}$ & $\longrightarrow$ & $\mathrm{H}_{2} \mathrm{O}+\mathrm{O}$ & $4.2 \cdot 10^{-12} \cdot \exp \left(\frac{-240}{T}\right)$ \\
\hline 14 & $\mathrm{H}+\mathrm{HO}_{2}$ & $\longrightarrow$ & $\mathrm{OH}+\mathrm{OH}$ & $6.6 \cdot 10^{-11}$ \\
\hline 15 & $\mathrm{H}+\mathrm{HO}_{2}$ & $\longrightarrow$ & $\mathrm{H}_{2}+\mathrm{O}_{2}$ & $1.5 \cdot 10^{-11}$ \\
\hline 16 & $\mathrm{H}+\mathrm{HO}_{2}$ & $\longrightarrow$ & $\mathrm{H}_{2} \mathrm{O}+\mathrm{O}$ & $1.6 \cdot 10^{-12}$ \\
\hline 17 & $\mathrm{NO}_{2}+\mathrm{O}$ & $\longrightarrow$ & $\mathrm{NO}+\mathrm{O}_{2}$ & $6.5 \cdot 10^{-12} \cdot \exp \left(\frac{120}{T}\right)$ \\
\hline 18 & $\mathrm{NO}+\mathrm{O}_{3}$ & $\longrightarrow$ & $\mathrm{NO}_{2}+\mathrm{O}_{2}$ & $2.0 \cdot 10^{-12} \cdot \exp \left(\frac{-1400}{T}\right)$ \\
\hline 19 & $\mathrm{NO}_{2}+\mathrm{O}_{3}$ & $\longrightarrow$ & $\mathrm{NO}_{3}+\mathrm{O}_{2}$ & $1.2 \cdot 10^{-13} \cdot \exp \left(\frac{-2450}{T}\right)$ \\
\hline 20 & $\mathrm{HNO}_{3}+\mathrm{OH}$ & $\longrightarrow$ & $\mathrm{H}_{2} \mathrm{O}+\mathrm{NO}_{3}$ & $7.2 \cdot 10^{-15} \cdot \exp \left(\frac{78 \frac{1}{T}}{T}\right)$ \\
\hline 21 & $\mathrm{NO}_{2}+\mathrm{OH}+M$ & $\longrightarrow$ & $\mathrm{HNO}_{3}+M$ & $k_{0}=2.5 \cdot 10^{-30}\left(\frac{300}{T}\right)^{4.4} \cdot k_{\infty}=1.6 \cdot 10^{-11} \cdot\left(\frac{300}{T}\right)^{1.7}$ \\
\hline 22 & $\mathrm{~N}_{2} \mathrm{O}+\mathrm{O}\left({ }^{1} \mathrm{D}\right)$ & $\longrightarrow$ & $\mathrm{NO}+\mathrm{NO}$ & $6.7 \cdot 10^{-11}$ \\
\hline 23 & $\mathrm{~N}_{2} \mathrm{O}+\mathrm{O}\left({ }^{1} \mathrm{D}\right)$ & $\longrightarrow$ & $\mathrm{N}_{2}+\mathrm{O}_{2}$ & $4.9 \cdot 10^{-11}$ \\
\hline 24 & $\mathrm{HO}_{2}+\mathrm{HO}_{2}$ & $\longrightarrow$ & $\mathrm{H}_{2} \mathrm{O}_{2}+\mathrm{O}_{2}$ & $2.3 \cdot 10^{-13} \cdot \exp \left(\frac{600}{T}\right)$ \\
\hline 25 & $\mathrm{OH}+\mathrm{H}_{2} \mathrm{O}_{2}$ & $\longrightarrow$ & $\mathrm{H}_{2} \mathrm{O}+\mathrm{HO}_{2}$ & $2.9 \cdot 10^{-12} \cdot \exp \left(\frac{-160}{T}\right)$ \\
\hline 26 & $\mathrm{CH}_{4}+\mathrm{OH}$ & $\longrightarrow$ & $\mathrm{CH}_{3}+\mathrm{H}_{2} \mathrm{O}$ & $2.4 \cdot 10^{-12} \cdot \exp \left(\frac{-1775}{T}\right)$ \\
\hline 27 & $\mathrm{O}\left({ }^{1} \mathrm{D}\right)+\mathrm{CH}_{4}$ & $\longrightarrow$ & $\mathrm{OH}+\mathrm{CH}_{3}$ & $1.5 \cdot 10^{-10}$ \\
\hline 28 & $\mathrm{O}\left({ }^{1} \mathrm{D}\right)+\mathrm{H}_{2}$ & $\longrightarrow$ & $\mathrm{OH}+\mathrm{H}$ & $1.1 \cdot 10^{-10}$ \\
\hline 29 & $\mathrm{OH}+\mathrm{CO}$ & $\longrightarrow$ & $\mathrm{CO}_{2}+\mathrm{H}$ & $1.5 \cdot 10^{-13}\left(1 .+0.6 \cdot\left(\frac{P}{1013}\right)\right.$ \\
\hline 30 & $\mathrm{HO}_{2}+\mathrm{NO}$ & $\longrightarrow$ & $\mathrm{NO}_{2}+\mathrm{OH}$ & $3.5 \cdot 10^{-12} \cdot \exp \left(\frac{250}{T}\right)$ \\
\hline 31 & $\mathrm{HO}_{2}+\mathrm{O}_{3}$ & $\longrightarrow$ & $\mathrm{OH}+2 \mathrm{O}_{2}$ & $1.1 \cdot 10^{-14} \cdot \exp \left(\frac{-500}{T}\right)$ \\
\hline 32 & $\mathrm{HO}_{2}+\mathrm{NO}_{2}+M$ & $\longrightarrow$ & $\mathrm{HO}_{2} \mathrm{NO}_{2}+M$ & $k_{0}=1.8 \cdot 10^{-31} \cdot\left(\frac{300}{T}\right)^{3.2} \cdot k_{\infty}=4.7 \cdot 10^{-12}\left(\frac{300}{T}\right)^{1.4}$ \\
\hline 33 & $\mathrm{HO}_{2} \mathrm{NO}_{2}+M$ & $\longrightarrow$ & $\mathrm{HO}_{2}+\mathrm{NO}_{2}+M$ & $k_{32} /\left(2.1 \cdot 10^{-27}\right) \cdot \exp \left(\frac{10900}{T}\right)$ \\
\hline 34 & $\mathrm{HO}_{2} \mathrm{NO}_{2}+\mathrm{OH}$ & $\longrightarrow$ & $\mathrm{H}_{2} \mathrm{O}+\mathrm{O}_{2}+\mathrm{NO}_{2}$ & $1.3 \cdot 10^{-12} \cdot \exp \left(\frac{380}{T}\right)$ \\
\hline 35 & $\mathrm{O}\left({ }^{1} \mathrm{D}\right)+\mathrm{CFCl}_{3}$ & $\longrightarrow$ & $3 \mathrm{Cl}+$ Products & $2.3 \cdot 10^{-10}$ \\
\hline 36 & $\mathrm{O}\left({ }^{1} \mathrm{D}\right)+\mathrm{CF}_{2} \mathrm{Cl}_{2}$ & $\longrightarrow$ & $2 \mathrm{Cl}+$ Products & $1.4 \cdot 10^{-10}$ \\
\hline 37 & $\mathrm{Cl}+\mathrm{O}_{3}$ & $\longrightarrow$ & $\mathrm{ClO}+\mathrm{O}_{2}$ & $2.9 \cdot 10^{-11} \cdot \exp \left(\frac{-260}{T}\right)$ \\
\hline 38 & $\mathrm{ClO}+\mathrm{O}$ & $\longrightarrow$ & $\mathrm{Cl}+\mathrm{O}_{2}$ & $3.0 \cdot 10^{-11} \cdot \exp \left(\frac{70}{T}\right)$ \\
\hline 39 & $\mathrm{Cl}+\mathrm{H}_{2} \mathrm{O}_{2}$ & $\longrightarrow$ & $\mathrm{HCl}+\mathrm{HO}_{2}$ & $1.1 \cdot 10^{-11} \cdot \exp \left(\frac{-980}{T}\right)$ \\
\hline 40 & $\mathrm{ClO}+\mathrm{NO}$ & $\longrightarrow$ & $\mathrm{Cl}+\mathrm{NO}_{2}$ & $6.4 \cdot 10^{-12} \cdot \exp \left(\frac{290}{T}\right)$ \\
\hline 41 & $\mathrm{Cl}+\mathrm{CH}_{4}$ & $\longrightarrow$ & $\mathrm{HCl}+\mathrm{CH}_{3}$ & $1.1 \cdot 10^{-11} \cdot \exp \left(\frac{-1400}{T}\right)$ \\
\hline 42 & $\mathrm{Cl}+\mathrm{H}_{2}$ & $\longrightarrow$ & $\mathrm{HCl}+\mathrm{H}$ & $3.7 \cdot 10^{-11} \cdot \exp \left(\frac{-2300}{T}\right)$ \\
\hline 43 & $\mathrm{Cl}+\mathrm{HO}_{2}$ & $\longrightarrow$ & $\mathrm{HCl}+\mathrm{O}_{2}$ & $1.8 \cdot 10^{-11} \cdot \exp \left(\frac{170}{T}\right)$ \\
\hline 44 & $\mathrm{Cl}+\mathrm{HO}_{2}$ & $\longrightarrow$ & $\mathrm{OH}+\mathrm{ClO}$ & $4.1 \cdot 10^{-11} \cdot \exp \left(\frac{-450}{T}\right)$ \\
\hline 45 & $\mathrm{HCl}+\mathrm{OH}$ & $\longrightarrow$ & $\mathrm{Cl}+\mathrm{H}_{2} \mathrm{O}$ & $2.6 \cdot 10^{-12} \cdot \exp \left(\frac{-350}{T}\right)$ \\
\hline
\end{tabular}




\begin{tabular}{|c|c|c|c|c|}
\hline No. & Reaction & & & Rate \\
\hline 46 & $\mathrm{ClO}+\mathrm{NO}_{2}+M$ & $\longrightarrow$ & $\mathrm{ClONO}_{2}+M$ & $k_{0}=1.8 \cdot 10^{-31} \cdot\left(\frac{300}{T}\right)^{3.4} \cdot k_{\infty}=1.5 \cdot 10^{-11}\left(\frac{300}{T}\right)^{1.9}$ \\
\hline 47 & $\mathrm{ClONO}_{2}+\mathrm{O}$ & $\longrightarrow$ & $\mathrm{ClO}+\mathrm{NO}_{3}$ & $2.9 \cdot 10^{-12} \cdot \exp \left(\frac{-800}{T}\right)$ \\
\hline 48 & $\mathrm{HOCl}+\mathrm{Cl}$ & $\longrightarrow$ & $\mathrm{HCl}+\mathrm{ClO}$ & $2.5 \cdot 10^{-12} \cdot \exp \left(\frac{-130}{T}\right)$ \\
\hline 49 & $\mathrm{ClO}+\mathrm{HO}_{2}$ & $\longrightarrow$ & $\mathrm{HOCl}+\mathrm{O}_{2}$ & $4.8 \cdot 10^{-13} \cdot \exp \left(\frac{700}{T}\right)$ \\
\hline 50 & $\mathrm{HOCl}+\mathrm{OH}$ & $\longrightarrow$ & $\mathrm{H}_{2} \mathrm{O}+\mathrm{ClO}$ & $3.0 \cdot 10^{-12} \cdot \exp \left(\frac{-500}{T}\right)$ \\
\hline 51 & $\mathrm{HOCl}+\mathrm{O}$ & $\longrightarrow$ & $\mathrm{OH}+\mathrm{ClO}$ & $1.7 \cdot 10^{-13}+1 /$ \\
\hline 52 & $\mathrm{ClO}+\mathrm{ClO}+M$ & $\longrightarrow$ & $\mathrm{Cl}_{2} \mathrm{O}_{2}+M$ & $k_{0}=2.2 \cdot 10^{-32} \cdot\left(\frac{300}{T}\right)^{3.1} \cdot k_{\infty}=3.5 \cdot 10^{-12}\left(\frac{300}{T}\right)^{1.0}$ \\
\hline 53 & $\mathrm{ClO}+\mathrm{ClO}$ & $\longrightarrow$ & $2 \mathrm{Cl}+\mathrm{O}_{2}$ & $1.2 \cdot 10^{-12} \cdot \exp \left(\frac{-1590}{T}\right)$ \\
\hline 54 & $\mathrm{ClO}+\mathrm{ClO}$ & $\longrightarrow$ & $\mathrm{Cl}+\mathrm{OClO}$ & $3.5 \cdot 10^{-13} \cdot \exp \left(\frac{-1370}{T}\right)$ \\
\hline 55 & $\mathrm{Cl}_{2} \mathrm{O}_{2}+M$ & $\longrightarrow$ & $\mathrm{ClO}+\mathrm{ClO}+M$ & $k_{52} / 3.0 \cdot 10^{-27} \cdot \exp \left(\frac{8450}{T}\right)$ \\
\hline 56 & $\mathrm{~N}+\mathrm{NO}$ & $\longrightarrow$ & $\mathrm{N}_{2}+\mathrm{O}$ & $2.1 \cdot 10^{-11} \cdot \exp \left(\frac{100}{T}\right)$ \\
\hline 57 & $\mathrm{~N}+\mathrm{O}_{2}$ & $\longrightarrow$ & $\mathrm{NO}+\mathrm{O}$ & $1.5 \cdot 10^{-11} \cdot \exp \left(\frac{-3600}{T}\right)$ \\
\hline 58 & $\mathrm{ClO}+\mathrm{OH}$ & $\longrightarrow$ & $\mathrm{Cl}+\mathrm{HO}_{2}$ & $1.1 \cdot 10^{-11} \cdot \exp \left(\frac{120}{T}\right)$ \\
\hline 59 & $\mathrm{OH}+\mathrm{CH}_{3} \mathrm{Cl}$ & $\longrightarrow$ & $\mathrm{Cl}+$ Products & $4.0 \cdot 10^{-12} \cdot \exp \left(\frac{-1400}{T}\right)$ \\
\hline 60 & $\mathrm{NO}_{2}+\mathrm{NO}_{3}+M$ & $\longrightarrow$ & $\mathrm{N}_{2} \mathrm{O}_{5}+M$ & $k_{0}=2.2 \cdot 10^{-30} \cdot\left(\frac{300}{T}\right)^{3.9} \cdot k_{\infty}=1.5 \cdot 10^{-12}\left(\frac{300}{T}\right)^{0.7}$ \\
\hline 61 & $\mathrm{~N}_{2} \mathrm{O}_{5}+M$ & $\longrightarrow$ & $\mathrm{NO}_{3}+\mathrm{NO}_{2}+M$ & $k_{60} / 4.0 \cdot 10^{-27} \cdot \exp \left(\frac{10930}{T}\right)$ \\
\hline 62 & $\mathrm{CH}_{3}+\mathrm{O}_{2}+M$ & $\longrightarrow$ & $\mathrm{CH}_{3} \mathrm{O}_{2}+M$ & $k_{0}=4.5 \cdot 10^{-31} \cdot\left(\frac{300}{T}\right)^{3.0} \cdot k_{\infty}=1.8 \cdot 10^{-12}\left(\frac{300}{T}\right)^{1.7}$ \\
\hline 63 & $\mathrm{CH}_{3} \mathrm{O}_{2}+\mathrm{NO}$ & $\longrightarrow$ & $\mathrm{CH}_{3} \mathrm{O}+\mathrm{NO}_{2}$ & $3.0 \cdot 10^{-12} \cdot \exp \left(\frac{280}{T}\right)^{1}$ \\
\hline 64 & $\mathrm{CH}_{3} \mathrm{O}_{2}+\mathrm{HO}_{2}$ & $\longrightarrow$ & $\mathrm{O}_{2}+$ Products & $3.8 \cdot 10^{-13} \cdot \exp \left(\frac{800}{T}\right)$ \\
\hline 65 & $\mathrm{CH}_{3} \mathrm{O}_{2}+\mathrm{CH}_{3} \mathrm{O}_{2}$ & $\longrightarrow$ & $2 \mathrm{CH}_{3} \mathrm{O}+\mathrm{O}_{2}$ & $2.5 \cdot 10^{-13} \cdot \exp \left(\frac{190}{T}\right)$ \\
\hline 66 & $\mathrm{CH}_{3} \mathrm{O}+\mathrm{O}_{2}$ & $\longrightarrow$ & $\mathrm{CH}_{2} \mathrm{O}+\mathrm{HO}_{2}$ & $3.9 \cdot 10^{-14} \cdot \exp \left(\frac{-900}{T}\right)$ \\
\hline 67 & $\mathrm{CH}_{2} \mathrm{O}+\mathrm{OH}$ & $\longrightarrow$ & $\mathrm{H}_{2} \mathrm{O}+$ Products & $1.0 \cdot 10^{-11}$ \\
\hline 68 & $\mathrm{CH}_{2} \mathrm{O}+\mathrm{O}$ & $\longrightarrow$ & $\mathrm{CHO}+\mathrm{OH}$ & $3.4 \cdot 10^{-11} \cdot \exp \left(\frac{-1600}{T}\right)$ \\
\hline 69 & $\mathrm{H}_{2}+\mathrm{OH}$ & $\longrightarrow$ & $\mathrm{H}_{2} \mathrm{O}+\mathrm{H}$ & $5.5 \cdot 10^{-12} \cdot \exp \left(\frac{-2000}{T}\right)$ \\
\hline 70 & $\mathrm{CH}_{2} \mathrm{O}+\mathrm{Cl}$ & $\longrightarrow$ & $\mathrm{CHO}+\mathrm{HCl}$ & $8.1 \cdot 10^{-11} \cdot \exp \left(\frac{-30}{T}\right)$ \\
\hline 71 & $\mathrm{O}\left({ }^{1} \mathrm{D}\right)+\mathrm{CH}_{4}$ & $\longrightarrow$ & $\mathrm{H}_{2}+\mathrm{CH}_{2} \mathrm{O}$ & $1.5 \cdot 10^{-10}$ \\
\hline 72 & $\mathrm{CH}_{3} \mathrm{O}_{2}+\mathrm{ClO}$ & $\longrightarrow$ & $\mathrm{CH}_{3} \mathrm{O}+\mathrm{Cl}+\mathrm{O}_{2}$ & $3.3 \cdot 10^{-12} \cdot \exp \left(\frac{-115}{T}\right)$ \\
\hline 73 & $\mathrm{Br}+\mathrm{O}_{3}$ & $\longrightarrow$ & $\mathrm{BrO}+\mathrm{O}_{2}$ & $1.7 \cdot 10^{-11} \cdot \exp \left(\frac{-800}{T}\right)$ \\
\hline 74 & $\mathrm{BrO}+\mathrm{O}$ & $\longrightarrow$ & $\mathrm{Br}+\mathrm{O}_{2}$ & $1.9 \cdot 10^{-11} \cdot \exp \left(\frac{230}{T}\right)$ \\
\hline 75 & $\mathrm{BrO}+\mathrm{NO}$ & $\longrightarrow$ & $\mathrm{Br}+\mathrm{NO}_{2}$ & $8.8 \cdot 10^{-12} \cdot \exp \left(\frac{260}{T}\right)$ \\
\hline 76 & $\mathrm{BrO}+\mathrm{OH}$ & $\longrightarrow$ & $\mathrm{Br}+\mathrm{HO}_{2}$ & $7.5 \cdot 10^{-11}$ \\
\hline 77 & $\mathrm{BrO}+\mathrm{ClO}$ & $\longrightarrow$ & $\mathrm{OClO}+\mathrm{Br}$ & $1.6 \cdot 10^{-12} \cdot \exp \left(\frac{430}{T}\right)$ \\
\hline 78 & $\mathrm{BrO}+\mathrm{ClO}$ & $\longrightarrow$ & $\mathrm{Br}+\mathrm{Cl}+\mathrm{O}_{2}$ & $2.9 \cdot 10^{-12} \cdot \exp \left(\frac{220}{T}\right)$ \\
\hline 79 & $\mathrm{BrO}+\mathrm{BrO}$ & $\longrightarrow$ & $2 \mathrm{Br}+\mathrm{O}_{2}$ & $1.5 \cdot 10^{-12} \cdot \exp \left(\frac{230}{T}\right)$ \\
\hline 80 & $\mathrm{BrO}+\mathrm{NO}_{2}+M$ & $\longrightarrow$ & $\mathrm{BrONO}_{2}+M$ & $k_{0}=5.2 \cdot 10^{-31} \cdot\left(\frac{300}{T}\right)^{3.2} \cdot k_{\infty}=6.9 \cdot 10^{-12}\left(\frac{300}{T}\right)^{2.9}$ \\
\hline 81 & $\mathrm{BrO}+\mathrm{HO}_{2}$ & $\longrightarrow$ & $\mathrm{HOBr}+\mathrm{O}_{2}$ & $3.4 \cdot 10^{-12} \cdot \exp \left(\frac{540}{T}\right)^{T}$ \\
\hline 82 & $\mathrm{O}+\mathrm{HOBr}$ & $\longrightarrow$ & $\mathrm{OH}+\mathrm{BrO}$ & $1.2 \cdot 10^{-10} \cdot \exp \left(\frac{-430}{T}\right)$ \\
\hline 83 & $\mathrm{CH}_{2} \mathrm{O}+\mathrm{Br}$ & $\longrightarrow$ & $\mathrm{HBr}+\mathrm{CHO}$ & $1.7 \cdot 10^{-11} \cdot \exp \left(\frac{-800}{T}\right)$ \\
\hline 84 & $\mathrm{Br}+\mathrm{HO}_{2}$ & $\longrightarrow$ & $\mathrm{HBr}+\mathrm{O}_{2}$ & $1.5 \cdot 10^{-11} \cdot \exp \left(\frac{-600}{T}\right)$ \\
\hline 85 & $\mathrm{HBr}+\mathrm{OH}$ & $\longrightarrow$ & $\mathrm{H}_{2} \mathrm{O}+\mathrm{Br}$ & $1.1 \cdot 10^{-11}$ \\
\hline 86 & $\mathrm{O}\left({ }^{1} \mathrm{D}\right)+\mathrm{CF}_{2} \mathrm{ClBr}$ & $\longrightarrow$ & $\mathrm{Br}+\mathrm{Cl}+\mathrm{Products}$ & $1.5 \cdot 10^{-10}$ \\
\hline 87 & $\mathrm{O}\left({ }^{1} \mathrm{D}\right)+\mathrm{CF}_{3} \mathrm{Br}$ & $\longrightarrow$ & $\mathrm{Br}+$ Products & $1.0 \cdot 10^{-10}$ \\
\hline 88 & $\mathrm{OH}+\mathrm{CH}_{3} \mathrm{Br}$ & $\longrightarrow$ & $\mathrm{Br}+$ Products & $4.0 \cdot 10^{-12} \cdot \exp \left(\frac{-1470}{T}\right)$ \\
\hline 89 & $\mathrm{O}\left({ }^{1} \mathrm{D}\right)+\mathrm{CH}_{3} \mathrm{Br}$ & $\longrightarrow$ & $\mathrm{Br}+$ Products & $1.8 \cdot 10^{-10}$ \\
\hline
\end{tabular}

$T$ is the temperature, $[M]$ is the atmospheric density $\left(\mathrm{cm}^{-3}\right)$ and $P$ is the pressure expressed in $\mathrm{hPa}$.

* Three-body reaction rates calculated with: $k([M], T)=k_{0}(T)[M] /\left[1+\frac{k_{0}(T)[M]}{k_{\infty}(T)}\right] \cdot 0.6^{\left\{1+\left(\log k_{0}(T)[M] / k_{\infty}(T)\right)^{2}\right\}^{-1}}$ 
Table 5. Reaction probabilities $(\gamma)$ for heterogeneous reactions

\begin{tabular}{lcll}
\hline & & & \\
Reaction & Liquid aerosols & NAT & ICE \\
\hline R1 & 0.1 & 0.0003 & 0.01 \\
R2 & - & 0.003 & 0.03 \\
R3 & $*^{1}$ & 0.001 & 0.1 \\
R4 & $*^{1}$ & 0.1 & 0.2 \\
R5 & $*^{2}$ & 0.1 & 0.3 \\
R6 & 0.4 & - & 0.3 \\
& & & \\
\hline
\end{tabular}

$*^{1}: \gamma$ values calculated on-line following Hanson and Ravishankara $(1994) ; *^{2}: \gamma$ value calculated on-line following Hanson et al. (1994)

calculated using the tabulated $\gamma$ values along with the surface particle area density and the mean kinetic speed of the reacting molecule in a gaseous phase (Rodriguez et al., 1989). Note that $\mathrm{Cl}_{2}$ and $\mathrm{ClNO}_{2}$ are assumed to exist only when produced through heterogeneous reactions and then, in the presence of sunlight, photodynamically converted into $\mathrm{ClO}_{x}$ and $\mathrm{NO}_{x}$ molecules. The particle surface area density is evaluated without introducing any detailed microphysics. The presence of NAT and ICE PSCs is predicted on the basis of simple thermodynamic considerations, as a function of temperature and $\mathrm{HNO}_{3}$ and $\mathrm{H}_{2} \mathrm{O}$ local partial pressure, respectively. These aerosol particles are typical of polar processes because they may grow at low temperatures (typically below $215 \mathrm{~K}$ ). NAT are assumed to form when they are thermodynamically possible, by evaluating the $\mathrm{HNO}_{3}$ saturation pressure calculated using the model temperature and mixing ratios of $\mathrm{H}_{2} \mathrm{O}$ and $\mathrm{HNO}_{3}$ with the algorithm of Hanson and Mauersberger (1988). Similarly, ICE PSCs are supposed to exist when $\mathrm{H}_{2} \mathrm{O}$ partial pressure is bigger than the saturation pressure of $\mathrm{H}_{2} \mathrm{O}$ over ice, evaluated using the Tetens equation (Murray, 1967). The excess of $\mathrm{H}_{2} \mathrm{O}$ and $\mathrm{HNO}_{3}$ is removed from the gas phase when saturation occurs and the available surface area density in the PSC region is estimated assuming a radius of 1 and $10 \mu \mathrm{m}$ for NAT and ICE, respectively, following Danilin et al. (1994). Sulfate aerosols are currently included using a zonal-mean aerosol climatology (WMO, 1991).

The model also includes a simple parameterization for sedimentation of PSC material and for the consequent denitrification and dehydratation effects on the stratosphere. Once they have been formed, NAT and ICE are sedimented with a mean speed of $15 \mathrm{~m} /$ day and $1.5 \mathrm{~km} /$ day, respectively, following Lefevre et al. (1994). Condensed species are then returned to the gas phase, depending on the ambient temperature, in the lower levels of the model where they have moved, affecting in such a way the vertical distribution of $\mathrm{HNO}_{3}$ and $\mathrm{H}_{2} \mathrm{O}$. At this stage $\mathrm{HCl}$ removal due to the incorporation into PSC particles has not been taken into account.

\subsection{Initialization}

Results of short time CTM integrations can depend critically on the initialization of the chemical fields. Our model can be initialized using zonally symmetrical tracer fields taken from two-dimensional models. In this case, following Douglass et al. (1990), long-lived tracer fields in the winter lower polar stratosphere are initially constrained to follow the morphology of the polar vortex using potential vorticity and potential temperature coordinate (conservative for adiabatic and frictionless motion) (e.g. see Redaelli et al., 1994, and references therein), while short-lived chemical species are brought into equilibrium with the 3-D initial meteorological fields performing a 2 day CTM integration with a constant temperature field and without transport (Chipperfield et al., 1993). Alternatively, full chemical output from another CTM long-term simulation, performed using analyzed winds and referred to the same period, can be used. Note that such a long-term simulation is not so strictly dependent on initialization conditions. When such fields are available, this can be the best choice, since both the 3-D long-lived and short-lived tracer fields are already constrained to the "real" meteorological situation from which we start our integration.

\section{Model experiment}

The STRATAQ CTM was integrated from 2 January until 20 March 1997. The UKMO data set was used for transport calculation, as described in Sect. 2.1. The model was initialized using output fields from long-term integration performed with the low resolution version of the 3-D SLIMCAT CTM (Chipperfield, 1999), relative to 2 January 1997, at 12:00 UTC. Winter 1996-97 has been chosen for its remarkable dynamical situation, along with the availability of sparse chlorine, nitrogen and $\mathrm{O}_{3}$ data, also showing very low $\mathrm{O}_{3}$ values in late March/April. The integration started in the period when minimum temperatures in the vortex started to show values below the PSC formation threshold, and continued to cover the ozone hole formation period, up to the dates when record minimum ozone column values where reported by the satellites.

The 1996-97 Northern Hemisphere polar vortex was very strong, as well as cold and symmetric, which is somewhat similar to what is usually found in the Antarctic spring vortex (Coy et al., 1997). Record low temperatures were set for most of March, and the minimum temperature remained below the type I PSC formation temperature from the middle of January through most of March. The later persistence of lower temperature possibly prolonged heterogeneous processing into a period of greater exposure to sunlight than in other years. In addition, the vortex remained substantially intact in the lower stratosphere into early May, exhibiting unprecedent longevity. Due to those unusual features, the winter 1996-97 is an excellent example of the impact of both dynamical and heterogeneous reactions on the chemistry of the stratosphere. 
Model outputs are shown here and compared with available global observations by MLS and TOMS instruments for selected days.

\section{Comparisons with observation}

\subsection{UARS MLS data}

The microwave Limb Sounder (MLS) on the UARS satellite made a measurement of $\mathrm{O}_{3}, \mathrm{ClO}$ and gas-phase $\mathrm{HNO}_{3}$ in the Arctic on selected days in December 1996, late January and February 1997 and April 1997 (Santee et al., 1997; Manney et al., 1997). Version 4 of the MLS gridded data obtained from the NASA/GSFC DAAC archive are used here, and stereographic maps relative to the $45 \mathrm{hPa}$ vertical level are shown, compared to the CTM data relative to the 11th model level, corresponding to $44.4 \mathrm{hPa}$. To examine data in light of the relative dynamical situation, UKMO temperatures at $50 \mathrm{hPa}$ are also shown for the chosen days in Fig. 1. We have overlaid on the temperature maps the 195 and $192 \mathrm{~K}$ temperature contours to approximately represent various PSC existence thresholds and also contours of UKMO isentropic PV at $475 \mathrm{~K}$, to indicate the approximate extent of the polar vortex.

Figure 2 shows model outputs for 28 January 1997, 26 days after the model initialization, corresponding to the resuming of MLS observation of northern high-latitudes. By that date, the stratospheric temperature at $475 \mathrm{~K}$ had been below $195 \mathrm{~K}$ for 25 days and below $192 \mathrm{~K}$ for 12 days (Coy et al., 1997), allowing for the formation of PSC particles and heterogeneous activation of chlorine. This is confirmed by the enhanced $\mathrm{ClO}$ present in the sunlit portion of the vortex in MLS. CTM local noon data satisfactorily reproduce the satellite $\mathrm{ClO}$ day time picture. However, features such as the three peak structure around $90 \mathrm{~W}$, present in $\mathrm{ClO}$, such as in $\mathrm{HNO}_{3}$ MLS data, are not reproduced in the CTM fields. In addition, CTM reports higher $\mathrm{ClO}$ data values if compared to MLS, but this should be primarily related to the use of local noon CTM fields in comparison to MLS data relative to the day side of the orbit. For this reason, CTM ClO local data should be considered as the maximum value expected for the selected day. Uniformly high $\mathrm{HNO}_{3}$ mixing ratios were observed inside the vortex by MLS, and reproduced by CTM. $\mathrm{HNO}_{3}$ capture by PSC is not active due to local temperatures that exceeded $195 \mathrm{~K}$, causing any PSC to disappear.

A different situation is shown in Fig. 3 for 24 February, (53 days after the CTMz initialization), where a large circumpolar zone of depleted gas-phase $\mathrm{HNO}_{3}$ is reported by MLS and well reproduced by CTM. This zone is strongly correlated with the inner part of the vortex with temperature values below $195 \mathrm{~K}$, where the model forms PSCs that capture $\mathrm{HNO}_{3}$ in solid phases. Significant MLS ClO enhancement extended over a large horizontal area, filling a larger portion of the vortex, as reproduced by the CTM field.

The CTM qualitatively reproduces the deactivation of active chlorine $\mathrm{ClO}_{x}\left(\mathrm{Cl}+\mathrm{ClO}+2 \mathrm{Cl}_{2} \mathrm{O}_{2}\right)$ and the formation dur-

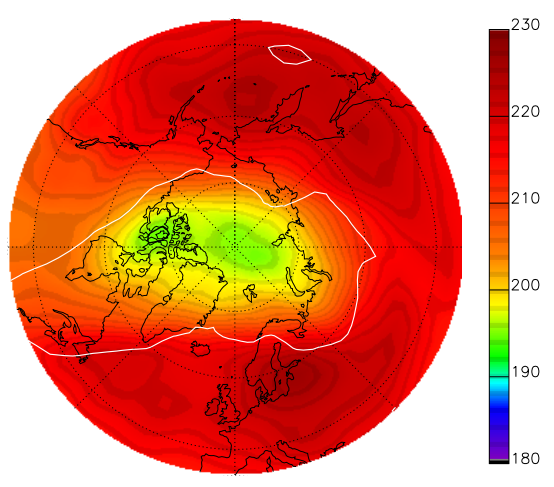

UKMO T - 970131

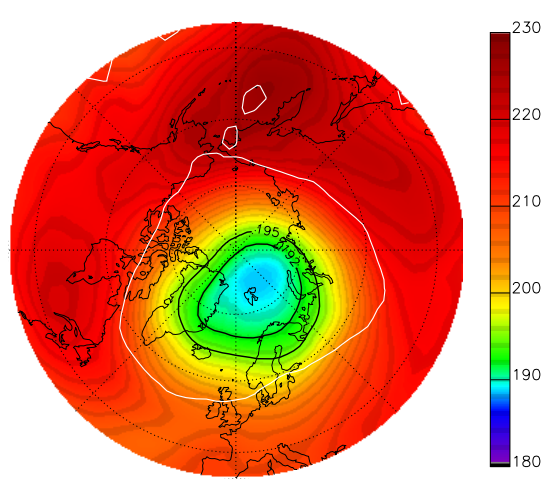

UKMO T - 970224

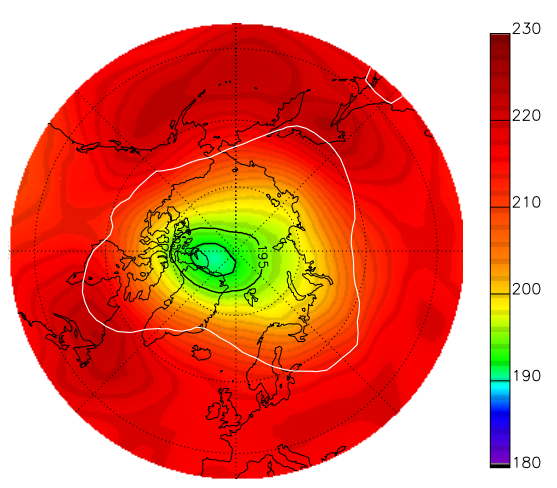

UKMO T - 970320

Fig. 1. False color maps of UKMO temperature at $50 \mathrm{hPa}$ at 12:00 UTC on (from top to bottom): 31 January, 24 February and 20 March 1997. Superimposed in black on the maps are the 195 and $192 \mathrm{~K}$ temperature contours to approximately represent various PSC existence thresholds. Superimposed in white are the $2.8 \cdot 10^{-4} \mathrm{Km}^{2} \mathrm{Kg}^{-1} \mathrm{~s}^{-1} \mathrm{UKMO}$ isentropic PV contours at $475 \mathrm{~K}$, as representative of the steepest PV gradient, to indicate the approximate edge of the vortex.

ing the spring period of $\mathrm{ClONO}_{2}$ removed during the winter period into the vortex region by heterogeneous reactions. Figure 4 shows CTM modeled fields for 24 February and 


\section{JANUARY 311997}

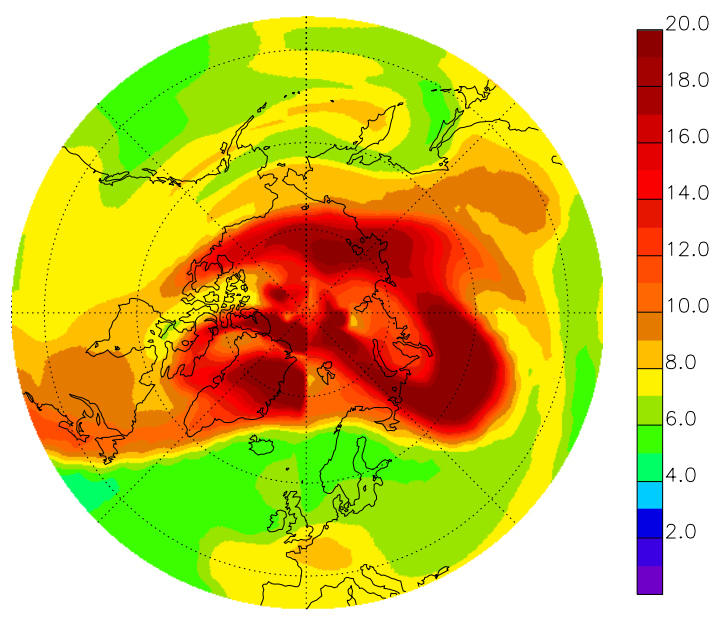

$\mathrm{CTM} \mathrm{HNO}_{3}$

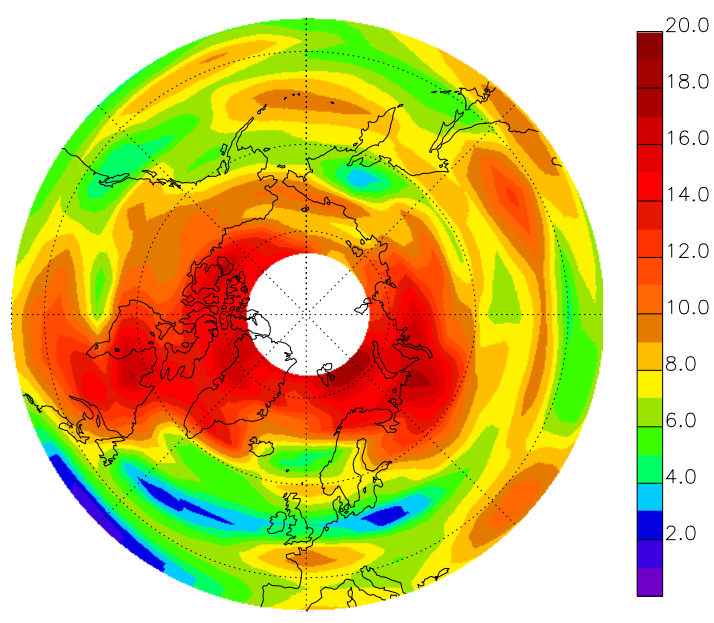

MLS $\mathrm{HNO}_{3}$

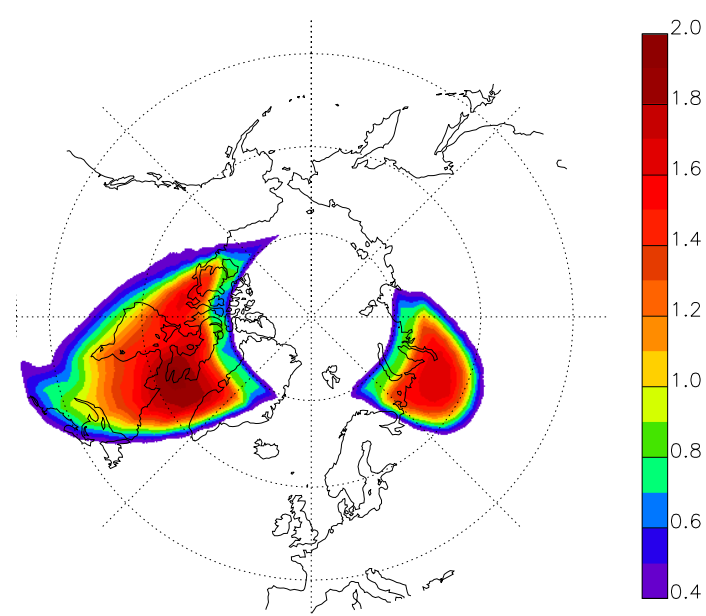

CTM $\mathrm{ClO}$

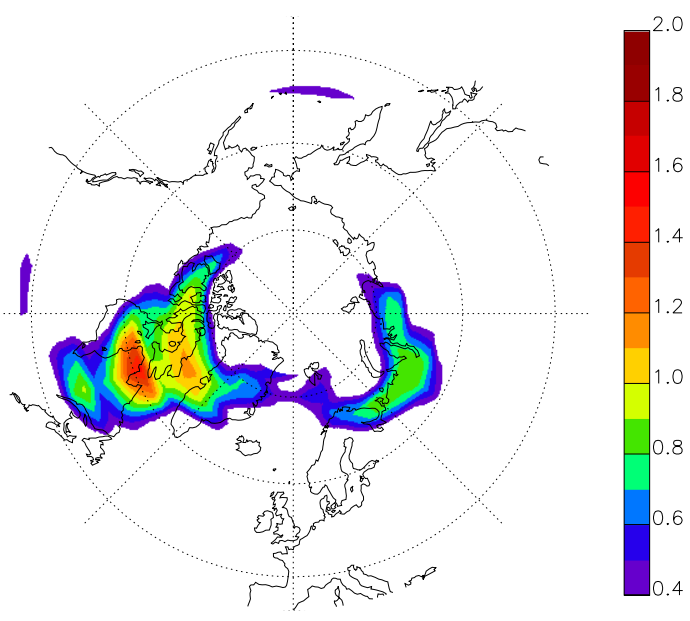

MLS ClO

Fig. 2. False color maps of $\mathrm{HNO}_{3}$ (left column) and $\mathrm{ClO}$ (right column) mixing ratio at about $45 \mathrm{hPa}$, on 31 January 1997 , from UARS/MLS data (bottom row) and as modelled with STRATAQ CTM (top row). Values are in ppbv. Only data for the dayside of the orbit are shown for MLS, while CTM data are plotted at local noon.

20 March 1997. In particluar, the CTM simulates the socalled $\mathrm{ClONO}_{2}$ "collar" (left column), which is repeatedly found in experimental data (e.g. Blom et al., 1995) and modeled by CTMs (e.g. Chipperfield et al., 1997), principally due to an in situ release of $\mathrm{NO}_{2}$ (e.g. from $\mathrm{HNO}_{3}$ ) that causes the gradual photochemical recovery of PSC processed air, leading the active chlorine in the air parcels to decay into $\mathrm{ClONO}_{2}$. While no satellite data are available for the chosen period for comparison, the values and structure of the modeled field seem to be consistent with literature data. In particular, the collar evolution is the one typical of the Northern Hemisphere, where the region of high $\mathrm{ClONO}_{2}$ at the vortex edge tends to extend and fill the whole vortex, with $\mathrm{ClONO}_{2}$ becoming the major chlorine species in late spring. A different recovery regime would be expected in the Southern Hemisphere (Douglass et al., 1995), where $\mathrm{ClO}$ in the vortex decays directly into $\mathrm{HCl}$, due to stronger denitrification. Figure 4 shows a $\mathrm{ClONO}_{2}$ collar, with mixing ratios in the range of about $0.5-2.0$ parts per billion in volume (ppbv), that seems to be bounded on the poleward side by regions not 


\section{FEBRUARY 241997}

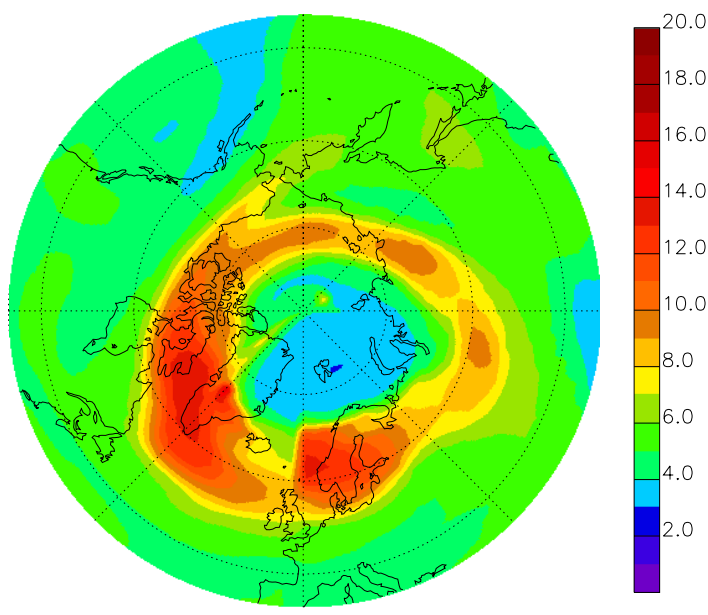

$\mathrm{CTM} \mathrm{HNO}_{3}$

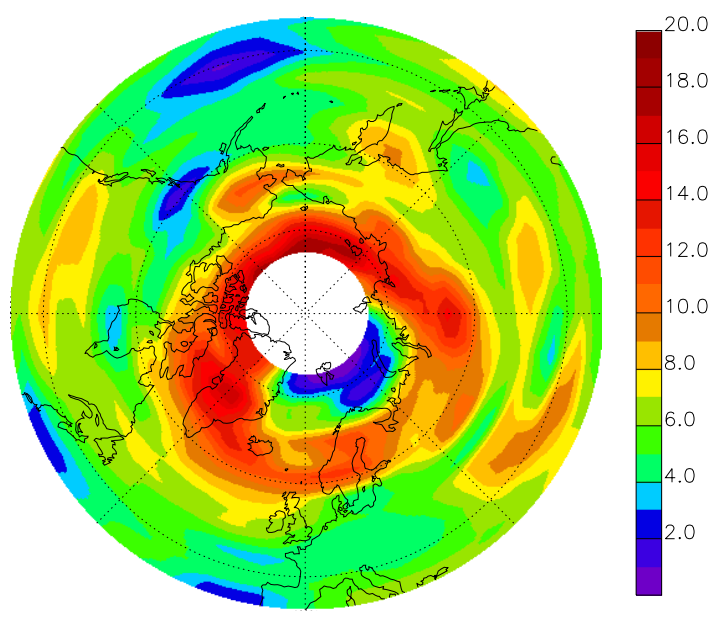

MLS $\mathrm{HNO}_{3}$

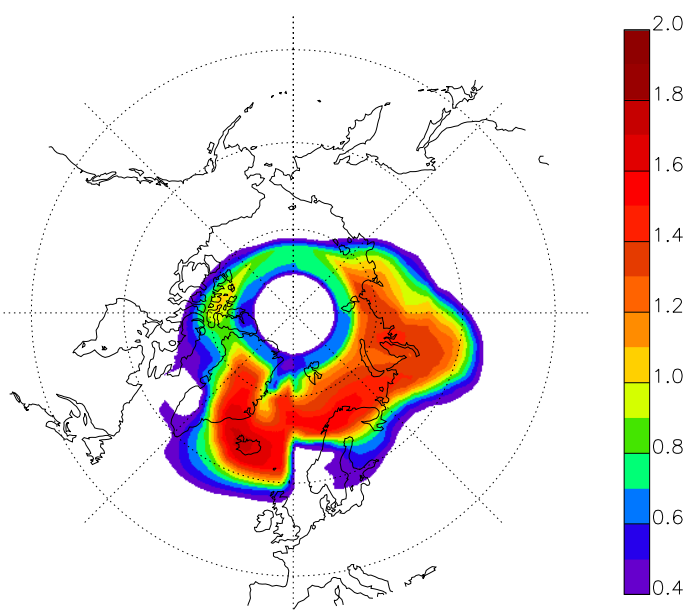

$\mathrm{CTM} \mathrm{ClO}$

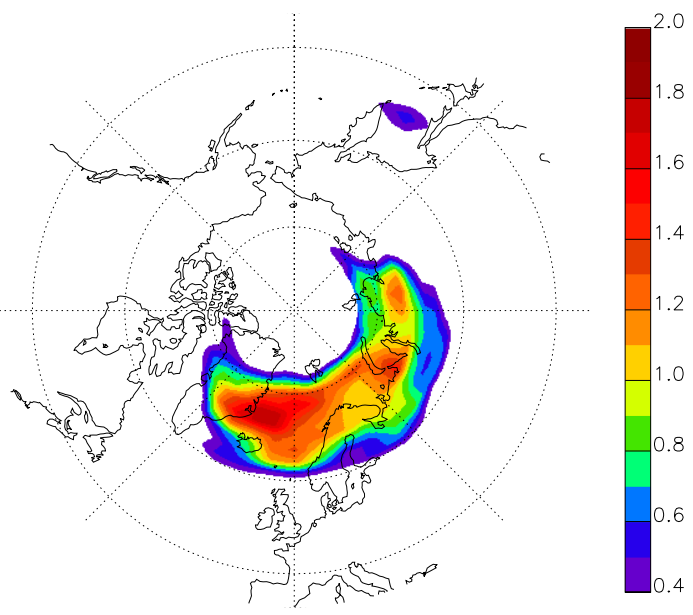

MLS ClO

Fig. 3. As in Fig. 2, but for 24 February 1997.

yet covered with sunlight and with temperatures cold enough for PSCs, and on the other side by the polar vortex border. Consistent with the theory, active chlorine $\mathrm{ClO}_{x}$ is strongly concentrated inside the collar, where photochemical recovery of PSC air, as described above, has not yet taken place, due to absence of sunlight.

\subsection{TOMS $\mathrm{O}_{3}$ columns}

In recent years, CTMs constrained by meteorological analysis have successfully reproduced the ozone column variability measured by TOMS (Douglass et al., 1996; Lefevre et al., 1998). Our CTM model output is compared with observations of the TOMS instrument on the NASDA/ADEOS spacecraft. Figure 5 compares a series of TOMS observations to STRATAQ CTM calculations, for different dates during the period covered by the run. Throughout the whole period the general synoptic features observable in the TOMS ozone column fields have a close correspondence with similar features in the CTM fields. Dynamically driven maxima and minima at middle latitudes are present in both the observed and calculated fields, showing an average hemispheric model/observation difference of about 0-25 DU. At vortex latitudes, CTM reproduces well the morphology and 


\section{FEBRUARY 241997}

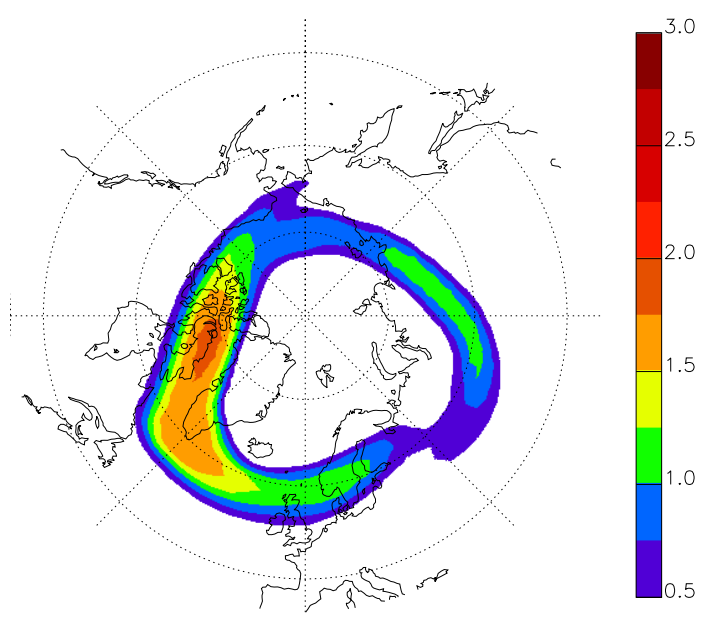

$\mathrm{CTM} \mathrm{ClONO}_{2}$

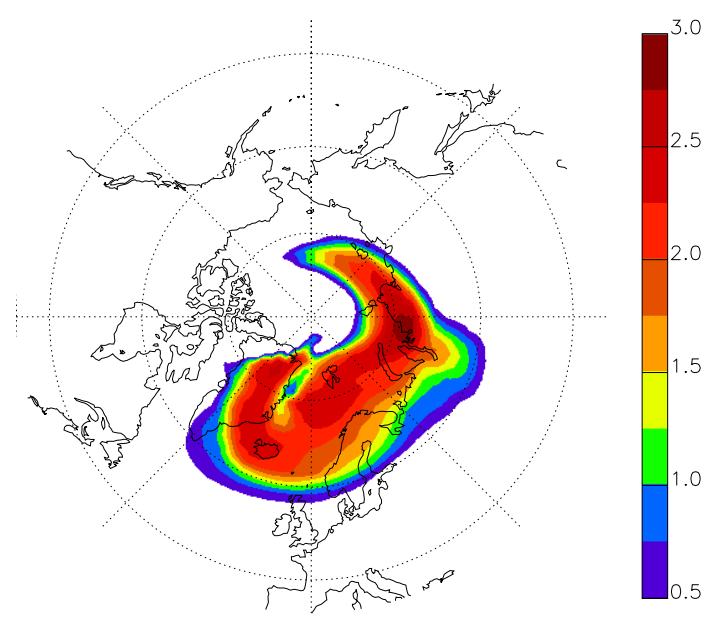

$\operatorname{CTM~} \mathrm{ClO}_{x}$

\section{MARCH 201997}

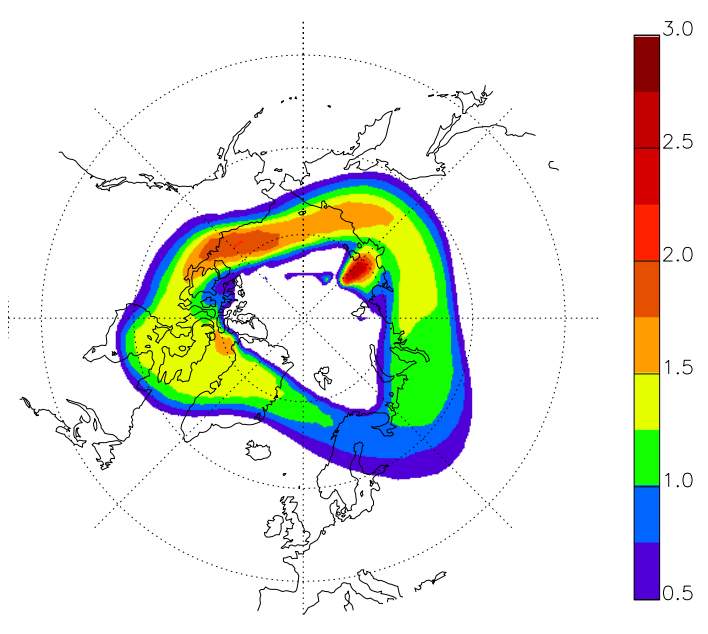

$\mathrm{CTM} \mathrm{ClONO}_{2}$

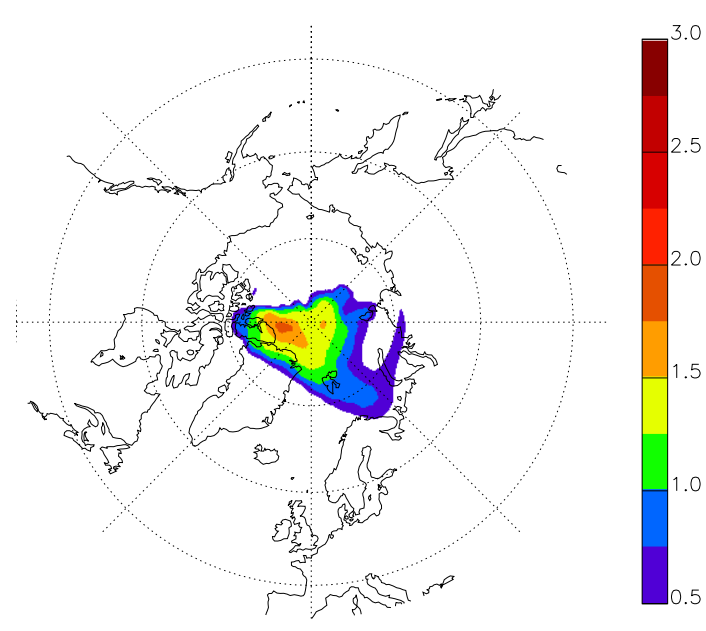

$\mathrm{CTM} \mathrm{ClO}_{x}$

Fig. 4. False color maps of $\mathrm{ClONO}_{2}$ (left column) and $\mathrm{ClO}_{x}$ (right column) mixing ratio at about $45 \mathrm{hPa}$, at 12:00 UTC on $24 \mathrm{February} 1997$ (top row) and on 20 March 1997 (bottom row) as modelled with STRATAQ CTM. Values are in ppbv.

partly the amplitude, of the continuous ozone decline, shown by TOMS during the investigated period. Starting from middle February, CTM shows a tendency to underestimate the $\mathrm{O}_{3}$ minimum centered on the North Pole, leading to model/observation differences of about $25-50 \mathrm{DU}$ in late March.
Such underestimation is rather suspect, especially when considering that the values of the $\mathrm{O}_{3}$ column measured by TOMS in the late 1997 arctic winter are by themselves already unusually low, and are believed to be the combined results not only of substantial photochemical loss, which is favored by high chlorine levels and long persisting cold 


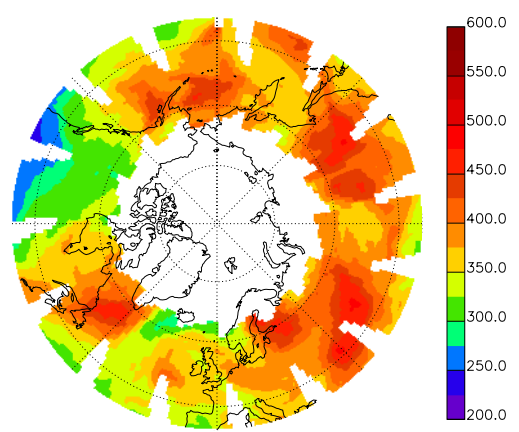

TOMS $\mathrm{O}_{3}-\mathbf{9 7 0 1 3 1}$

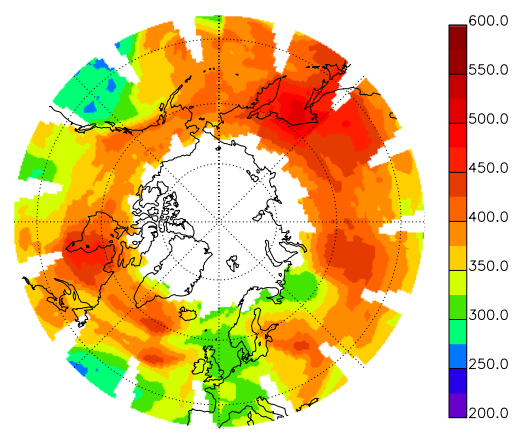

TOMS $\mathrm{O}_{3}-\mathbf{9 7 0 2 1 2}$

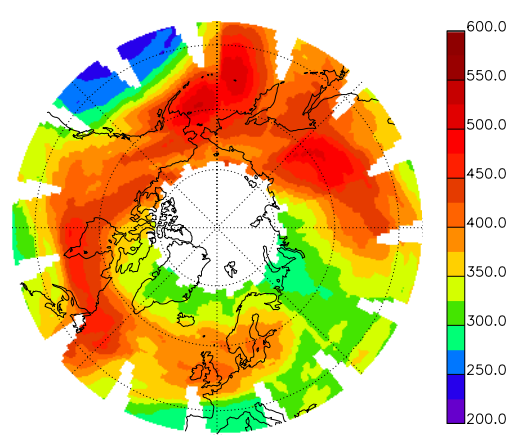

TOMS $\mathrm{O}_{3}-\mathbf{9 7 0 2 2 6}$

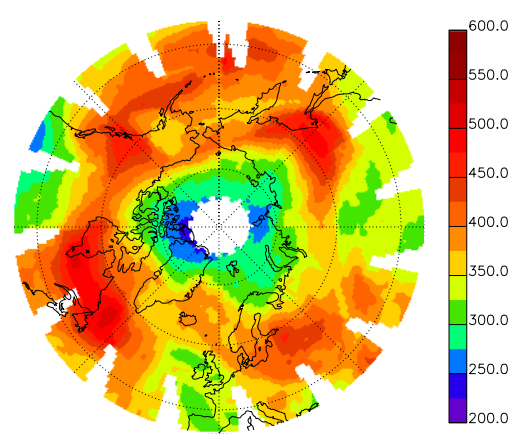

TOMS $\mathrm{O}_{3}-\mathbf{9 7 0 3 2 0}$

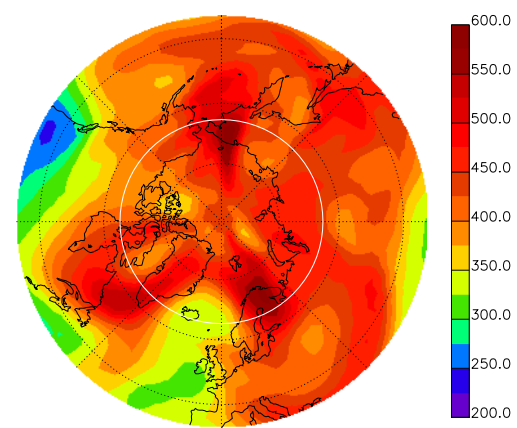

CTM $\mathrm{O}_{3}-\mathbf{9 7 0 1 3 1}$

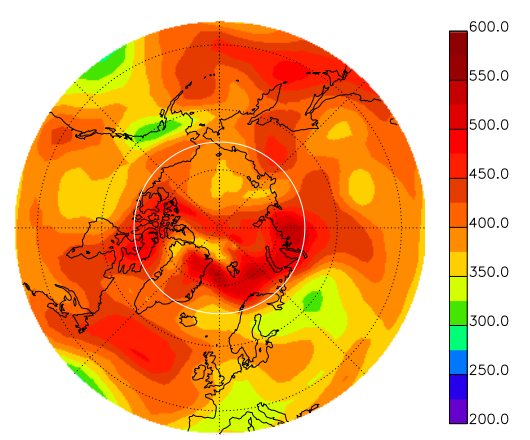

$\mathrm{CTM} \mathrm{O}_{3}-\mathbf{9 7 0 2 1 2}$

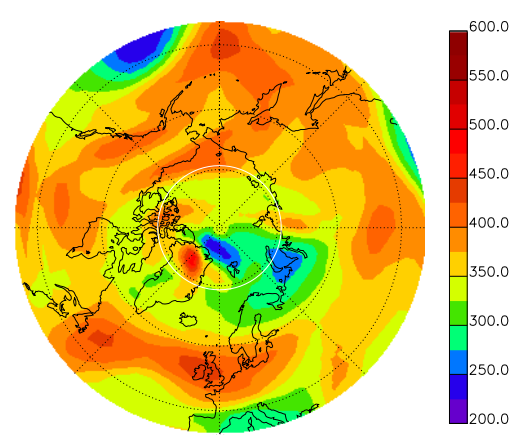

$\mathrm{CTM} \mathrm{O}_{3}-\mathbf{9 7 0 2 2 6}$

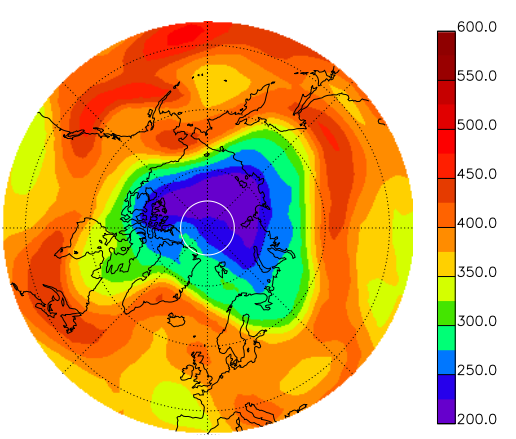

$\mathrm{CTM} \mathrm{O}_{3}-\mathbf{9 7 0 3 2 0}$
Fig. 5. False color maps of total ozone distribution (Dobson Units) measured by TOMS (left column) and modelled with STRATAQ CTM (right column), on 31 January, 12 February, 26 February, 20 March 1997. CTM data are for 12:00 UTC while TOMS data are asynoptic fields obtained near local noon. Superimposed in white are the latitude circles that approximately correspond to the polar zone where the TOMS data are missing due to an absence of solar light. 
temperatures, but also by of a large-scale dynamicallyinduced reduction linked to an anticyclonic disturbance that developed near the pole in the upper troposphere during March (Lefevre et al., 1998).

\section{Discussion}

From the examination of the $\mathrm{O}_{3}$ data shown, it seems that during the progress of the winter, as the $\mathrm{O}_{3}$ losses increases, so does the amplitude of the $\mathrm{O}_{3}$ column difference, with the model underestimating the $\mathrm{O}_{3}$ column value in the polar vortex in late March. We can suggest no certain cause for this behaviour, but some speculation can be made. Since the low $\mathrm{O}_{3}$ column values recorded in March are closely linked both to chemical and dynamical effects (Lefevre et al., 1998), the disagreement between the model and the observations during late winter can be due to an inadequacy of the model in correctly reproducing any of these effects.

To quantify chemical ozone loss we can consider the relationship between $\mathrm{O}_{3}$ and a practically inert tracer, $\mathrm{CH}_{4}$ (Proffitt et al., 1993; Muller et al., 1997a). Inside the vortex, this relation should not be altered by transport processes due to the limited exchange of air between the vortex and midlatitudes, and should be preserved throughout the lifetime of the vortex in absence of chemical processes. Thus, any deviation from the initial (i.e. corresponding to late November) $\mathrm{CH}_{4} / \mathrm{O}_{3}$ relationship over the winter must be due to chemical changes. Figure 6 shows this relationship between $\mathrm{CH}_{4}$ and $\mathrm{O}_{3}$ volume mixing ratios in the Northern Hemisphere stratosphere for the winter 1996-1997, as modelled by STRATAQ, by considering grid points inside the vortex and in the potential temperature range of 400-650 K. In comparing CTM data for November 1996 (corresponding to "early" vortex unperturbed conditions and obtained with a 10 day CTM run) with CTM data from March 1997 (corresponding to a "late" vortex period), ozone reductions are evident through all the height ranges considered. In particular, the model simulates chemical destruction resulting in local ozone losses of up to $85 \%$ over the potential temperature range of $450-500 \mathrm{~K}$, corresponding approximately to a $\mathrm{CH}_{4}$ concentration in the range of 0.6-1.0 ppmv. To estimate the ozone mixing ratio, which is expected in the absence of the chemical removal of ozone, we use the $\mathrm{CH}_{4} / \mathrm{O}_{3}$ relationship in the "early" vortex to obtain a proxy for the ozone mixing ratio $\mathrm{O}_{3}=f\left(\mathrm{CH}_{4}\right)$ that would be expected in the vortex if chemical processes were absent (Muller et al., 1997b). Such an $\mathrm{O}_{3}$ proxy, derived from a polynomial fit to November data, can be compared to $\mathrm{CTM} \mathrm{O}_{3}$ fields in late spring, to identify chemically removed $\mathrm{O}_{3}$ reproduced by CTM despite variations caused by dynamical processes. The accumulated $\mathrm{O}_{3}$ reduction between 30 November 1996 and 20 March 1997 is then integrated over the potential temperature range of 400 to $650 \mathrm{~K}$ to estimate the depletion in column ozone. The resulting ozone column loss reflects variability inside the vortex, showing chemical ozone losses up to 80-90 dobson units. Such values are about $10 \%$ higher than those derived for the same period
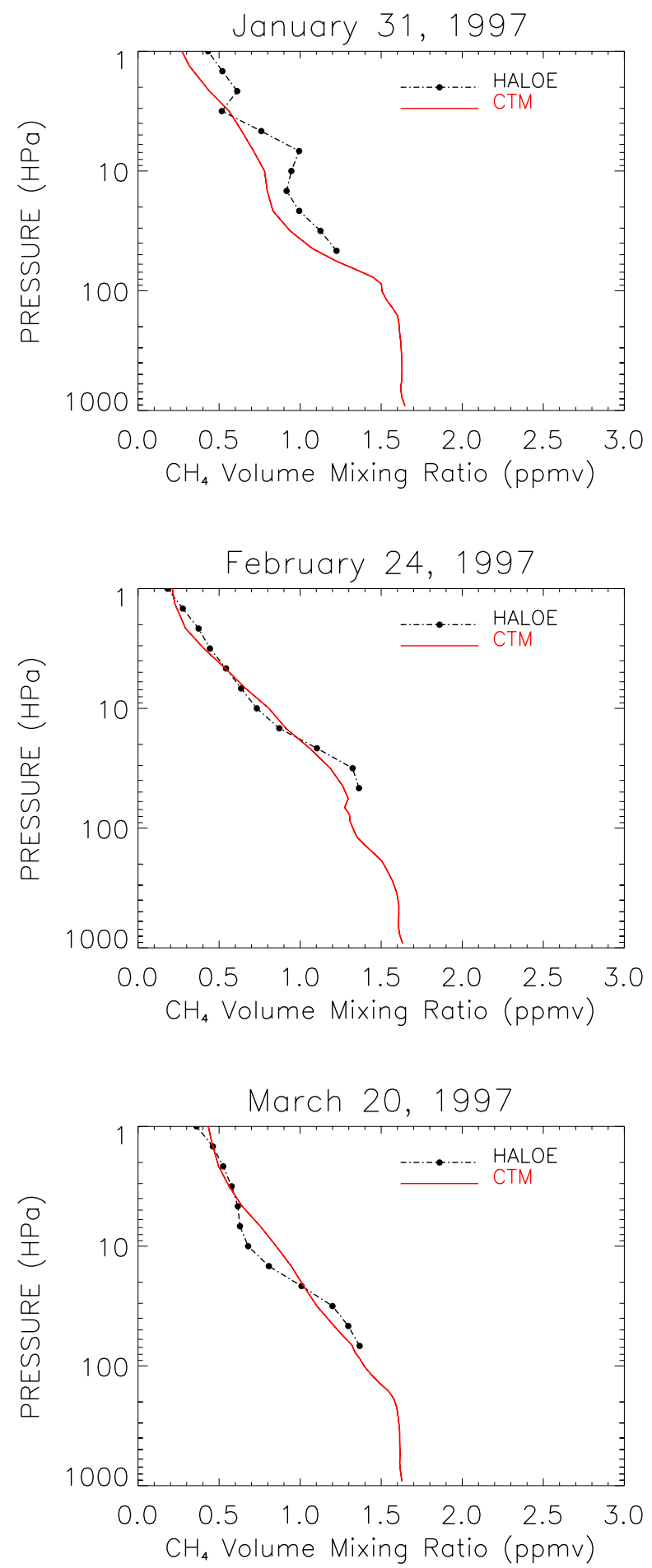

Fig. 6. $\mathrm{CH}_{4}$ profiles observed by HALOE and modeled with CTM for (from top to bottom): 31 January 1997 at $276^{\circ} \mathrm{E}$ and $35^{\circ} \mathrm{N}$; 24 February 1997 at $275^{\circ} \mathrm{E}$ and $47^{\circ} \mathrm{N} ; 20$ March 1997 at $259^{\circ} \mathrm{E}$ and $48^{\circ} \mathrm{N}$

by Muller et al. (1997b), using HALOE observations. However, such a discrepancy in the chemical contribution to the $\mathrm{O}_{3}$ column reduction cannot account by itself alone for the discrepancy found between TOMS and CTM column values 
for 20 March 1997 (see Fig. 5).

This result, together with the rather good performance of the model in reproducing, the timing and the extent of chlorine activation due to PSC air processing, as deducted from model to data comparison, even without a very detailed microphysical parameterization, suggests that we should concentrate instead on the possible inadequacy of the model dynamic. For example, the model description of horizontal and vertical transport could be in error. However, the model, forced by meteorological analyses and including an accurate and mass-conserving dynamical scheme, seems to reproduce fairly well the observed horizontal structure of $\mathrm{ClO}$ and $\mathrm{HNO}_{3}$ for the studied cases in strong correlation with the corresponding large-scale dynamical and meteorological situation (i.e. the position and the extent of the temperature minima and of the polar vortex). A significant systematic error in horizontal transport seems to be at least unlikely. An error in the vertical transport could instead explain part of the discrepancy. In particular, diabatic downward transport in the model that is to weak would cause a lower mixing ratio $\mathrm{O}_{3}$ to be carried from higher altitudes, leading to an underestimation of column ozone values and ozone in the lower stratosphere. Evidence for this could be added by validating the behaviour of the model $\mathrm{CH}_{4}$, i.e. treated almost as an inert tracer in the CTM. Figure shows profile comparisons between the CTM and the UARS Halogen Occultation Experiment (HALOE) $\mathrm{CH}_{4}$ data, for 31 January, 24 February and 20 March 1997, at middle latitudes. Unfortunately, no vertical profiles of $\mathrm{CH}_{4}$ are available inside the vortex for the studied winter period, due to the UARS orbit (Muller et al., 1997b). There is a clear difference between the various comparisons, with the model first underestimating, at almost every given altitudes, the observed $\mathrm{CH}_{4}$ profiles at the end of January, while showing reduced differences in late February, and an overestimation in late March, between 5 and $50 \mathrm{hPa}$. This may indicate a clear systematic tendency of the model to underestimate the vertical downward transport, although other causes cannot be excluded. In order to check this hypothesis better, the model output would need to be systematically compared with a great number of observations of longlived tracers, measured at adequate times and positions, and with good vertical resolution and coverage. No such data are available, at least for the studied winter. However, a general estimate of the vertical transport simulated by CTM can be obtained by examining directly the vertical velocity values as calculated by the model's internal transport code. Average vertical velocity values in the CTM, inside the vortex area on the $50 \mathrm{hPa}$ level, are of about $-0.1 \mathrm{~cm} / \mathrm{s}$ over the JanuaryFebruary period, consistent with data from literature (e.g. see Proffitt et al., 1993). But values for March show a small average upward motion component inside the vortex, which is quite unlikely during such a winter period. This outcome clearly shows that vertical velocity is a crucial factor in our model that deserves further studies.

In addition to dynamical errors, there could be of course other possible causes for the identified data/observation discrepancies, each with different and cumulative contributions

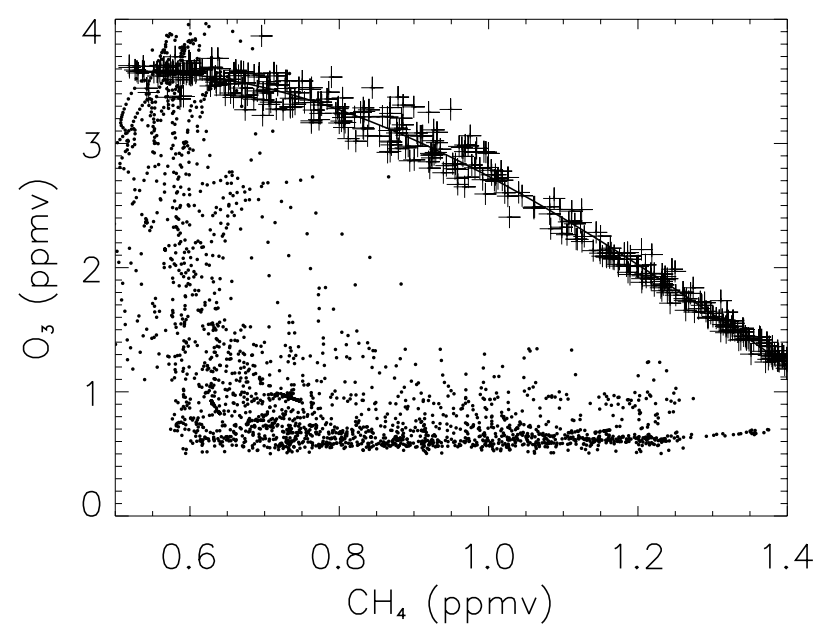

Fig. 7. Relationship between $\mathrm{CH}_{4}$ and $\mathrm{O}_{3}$ volume mixing ratios in the Northern Hemisphere stratosphere for the winter 1996-1997 as modelled by STRATAQ CTM. The data encompass potential temperature ranges from $400 \mathrm{~K}$ to $650 \mathrm{~K}$, but exclude points outside the vortex. Plus signs indicate CTM data for 30 November 1996, while black dots indicate CTM data for 20 March 1997. The black solid line shows the relation derived from the November data.

to the resulting fields. For example, we may suspect an incorrect estimation of the tropospheric contribution to the $\mathrm{O}_{3}$ total column, likely to be caused by the poor details in the tropospheric chemistry that can be of particular importance in the peculiar meteorological situation of the late 1997 winter, showing low temperature in the stratosphere, which is frequently linked to high tropopause. Clearly, further work is needed to resolve this issue.

\section{Summary}

STRATAQ is a global three-dimensional chemical and transport model of the stratosphere suitable for short-term integration to study transport and chemical evolution related to particular meteorological conditions in a given region of the stratosphere. The model includes detailed stratospheric chemistry of $\mathrm{O}_{x}, \mathrm{NO}_{y}, \mathrm{HO}_{x}, \mathrm{ClO}_{y}$ and $\mathrm{BrO}_{y}$, described with 89 homogeneous and 6 heterogeneous chemical reactions, 33 photochemical reactions, and provides the distribution of 43 chemical species. The behaviour of species is represented by the continuity and transport equation which includes the contribution of transport by the large-scale circulation and the tendency due to the chemistry. The continuity equations of the chemical species are solved numerically on a $5^{\circ}$ longitude $\times 2^{\circ}$ latitude horizontal grid and over $28 \sigma$ levels. The transport is calculated using a Van Leer-type scheme in a semi-lagrangian flux form. The chemical scheme is written for chemical families and a semi-implicit symmetric method is used to integrate the chemical equation. A state-of-theart model has been described and the results from the arctic winter 1996/97 simulation have been shown and compared to satellite data. 
Acknowledgement. We are grateful to $\mathrm{R}$. Rood for providing the Van Leer transport code, and to S. J. Lin and S. Steenrood for helping in its use. We are grateful to M. P. Chipperfield for kindly providing SLIMCAT CTM data used for the initialization. We would like to thank the Upper Atmosphere Research Satellite (UARS) Project, (Code 916), and the Distributed Active Archive Center (Code 902.2) at the Goddard Space Flight Center, Greenbelt, MD 20771 for the production and distribution of HALOE and MLS data. We thank the NASA/GSFC TOMS team for provision of TOMS data and the British Atmospheric Data Centre for provision of UKMO analyses.

Topical Editor J.-P. Duval thanks V. H. Peuch and another referee for their help in evaluating this paper.

\section{References}

Allen, D. J., Douglass, A. R., Rood, R. B. et al.: Application of a Monotonic Upstream-biased Transport Scheme to ThreeDimensional Constituent Transport Calculations, Mon. Weather Rev., 119, 2456-2464, 1991.

Allen, M. and Frederick, J. E.: Effective photo-dissociation cross sections for molecular oxygen and nitric oxide in the SchumannRunge bands, J. Atmos. Sci., 39, 2066-2073, 1982.

Austin, J., Knight, J., and Butchart, N.: Three-dimensional chemical model simulations of the ozone layer: 1979-2015, Q. J. R. Meteorol. Soc., 126, 1533-1556, 2000.

Austin, J., Butchart, N., and Knight. J.: Three-dimensional chemical model simulations of the ozone layer: 2015-55, Q. J. R. Meteorol. Soc., 127, 959-974, 2001.

Blom, C. E., Fischer, H., Glatthor, N., Gulde, T., Hopfner, M., and Piesch, C.: Spatial abd temporal variability of $\mathrm{ClONO}_{2}, \mathrm{HNO}_{3}$, and $\mathrm{O}_{3}$ in the Arctic winter of 1992/1993 as obtained by airborne infrared emission spectroscopy, J. Geophys. Res., 100, 91019114, 1995.

Brasseur, G. P., Hitchman, M. H., Walters, S., Dymek, M., Falise, E., and Pirre, M.: An interactive chemical-dynamical radiative two-dimensional model of the middle atmosphere, J. Geophys. Res., 95, 5639-5655, 1990.

Carli, B., Cortesi, U., Blom, C. E., Chipperfield, M. P., De Rossi, G., and Redaelli, G.: Airborne Polar Experiment Geophysica Aircraft in Antarctica (APE-GAIA), SPARC newsletter, 15, July 2000.

Chameides, W. L. and Cicerone, R. J.: Effects of non-methane hydrocarbons in the atmosphere, J. Geophys. Res., 83, 947-952, 1978.

Chipperfield, M. P., Cariolle, D., Simon, P., Ramaroson, R., and Lary, D. J.: A three-dimensional modeling study of trace species in the Arctic lower stratosphere during winter 1989-1990, J. Geophys. Res., 98, 7199-7218, 1993.

Chipperfield, M. P., Lutman, E. R., Kettlebotough, J. A., and Pyle, J. A.: Model studies of chlorine deactivation and formation of $\mathrm{ClONO}_{2}$ collar in the Arctic polar vortex, J. Geophys. Res., 102, 1467-1478, 1997.

Chipperfield, M. P.: Multiannual simulations with a threedimensional chemical transport model, J. Geophys. Res., 104, 1781-1805, 1999.

Coy, L., Nash, E. R., Newman, P. A.: Meteorology of the polar Vortex: Spring 1997, Geophys. Res. Lett., 24, 2693-2696, 1997.

Cunnold, D., Alyea, F., Phillips, N., and Prinn, R.: A Three-Dimensional Dynamical-Chemical Model of Atmospheric Ozone, J. Atmos. Sci., 32, 170-194, 1975.
Danilin, M. Y. and McConnel, J. C.: Heterogeneous reactions in a stratospheric box model: a sensitive study, J. Geophys. Res., 99, 25 681-25 696, 1994.

DeMore, W. B., Sander, S. P., Golden, D. M., et al.: Chemical Kinetics and Photochemical Data for Use in Stratospheric Modeling, JPL publication, 94-26, JPL, Pasadena, CA, 1994.

DeMore, W. B., Sander, S. P., Golden, D. M., et al.: Chemical Kinetics and Photochemical Data for Use in Stratospheric Modeling, JPL publication, 97-4, JPL, Pasadena, CA, 1997.

Douglass, A. R., Rood, R. B., Stolarski, R. S., et al.: Global threedimensional constituent fields derived from profile data, Geophys. Res. Lett., 17, 525-528, 1990.

Douglass, A. R, Schoeberl, M. R., Stolarski, R. S., Waters, J. W., Russel, J. M., Roche, A. E., and Massie, S. T.: Interhemispheric differences in springtime deactivation of vortex $\mathrm{ClO}$, J. Geophys. Res., 100, 13 967-13 978, 1995.

Douglass, A. R., Weaver, C. J., Rood, R. B., and Lawrence, C.: A three dimensional simulation of the ozone annual cycle using winds from a data assimilation system, J. Geophys. Res., 101, 1463-1474, 1996.

Hanson, D. and Mauersberger, K.: Laboratory studies of the nitric acid trihydrate: implications for the south polar stratosphere, Geophys. Res. Lett., 15, 855-858, 1988.

Hanson, D. R. and Ravishankara, A. R.: Reactive uptake of $\mathrm{ClONO}_{2}$ onto sulfuric acid due to reaction with $\mathrm{HCl}$ and $\mathrm{H}_{2} \mathrm{O}$, J. Phys. Chem., 98, 5728-5735, 1994.

Hanson, D. R., Ravishankara, A. R., and Salomon, S.: Heterogeneous reactions in sulfuric acid aerosols: A framework for model calculations, J. Geophys. Res., 99, 3615-3629, 1994.

Hein, R., Dameris, M., Schnadt, C., Land, C., Grewe, V., Kohler, I., Ponater, M., Sausen, R., Steil, B., Landgraf, J., and Bruhl, C.: Results of an interactively coupled atmospheric chemistrygeneral circulation model: 0 observations, Ann. Geophysicae, 19, 435-457, 2001.

Joseph, J. H., Wiscombe, W. J., and Weinman, J. A.: The delta Eddington approximation for radiative flux transfer J. Atmos. Sci., 33, 2452-2459, 1976.

Kawa, S. R., Fahey, D. W., Wilson, J. C., et al.: Interpretation of $\mathrm{NO}_{x} \mathrm{NO}_{y}$ observations from AASE-II using a model of chemistry along trajectories, Geophys. Res. Lett., 20, 2507-2510, 1993.

Ko, K. W., Schneider, H. R., Shia, R.-L., Weisenstein, D. K., and Sze, N.-D.: A two-dimensional model with coupled dynamics, radiation, and photo-chemistry, 1, Simulation of the middle atmosphere, J. Geophys. Res., 98, 20 429-20 440, 1993.

Lefevre, F., Brasseur, G. P., Folkins, I., Smith, A. K., and Simon, P.: Chemistry of the 1991-1992 stratospheric winter: threedimensional model simulations, J. Geophys. Res., 99, 81838195, 1994.

Lefevre, F., Figarol, F., Carslaw, K. S., and Peter, T.: The 1997 Arctic ozone depletion quantified from three-dimensional simulations, Geophys. Res. Lett., 25, 2425-2428, 1998.

Lin, S. J. and Rood, R. B.: Multidimensional Flux-Form SemiLagrangian Transport Schemes, Mon. Wheather Rev., 124 2046-2070, 1996.

MacKenzie, R., Santacesaria, V., Adriani, A., et al.: APE-THESEO: Clouds, dehydration, and transport at the tropical tropopause, SPARC newsletter, 14, January 2000.

Manney, G. L., Froidevaux, L., Santee, M. L., Zurek, R. W. and Waters, J. W.: MLS observations of Arctic ozone loss in 199697, Geophys. Res. Lett., 24, 2697-2700, 1997.

Müller, R., Crutzen, P. J., Grooss, J. U., et al.: Severe chemical 
ozone loss in the Arctic during the winter of 1995-96, Nature, 389, 709-712, 1997a.

Müller, R., Grooss, J. U., McKenna, D. S., et al.: HALOE observations of the vertical structure of chemical ozone depletion in the Arctic vortex during winter and early spring 1996-1997, Geophys. Res. Lett., 24, 2717-2720, 1997b.

Murray, F. W.: On the computation of saturation vapor pressure, J. Appl. Met., 6, 203-204, 1967.

Oreskes, N., Shrader-Frechette, K., and Belitz, K.: Verification, validation and confirmation of Numerical Models in the Earth Sciences, Science, 263, 641-646, 1994.

Pitari, G., Grassi, B., and Visconti, G.: Results of a Chemical Transport Model with Interactive Aerosol Microphysics, in: Proc. Of Quadrennial Ozone Symposium, L”Aquila, Italy, (Eds) Bojkov, R. and Visconti, G., 759-762, 1998.

Proffitt, M. H., Aikin, K., Margitan, J. J., et al.: Ozone Loss Inside the Northern Polar Vortex During the 1991-1992 Winter, Science, 261, 1150-1154, 1993.

Ramaroson, R., Pirre, M., and Cariolle, D.: A box model for online computations of diurnal variations in a 1-D model: potential for application in multidimensional cases, Ann. Geophysicae, 10, 416-428, 1992.

Redaelli, G., Lait, L., Schoeberl, M., Newman, P. A., Visconti, G., D’Altorio, A., Masci, F., Rizi, V., Froidevaux, L., Waters, J. W., and Miller, A. J.: UARS MLS $\mathrm{O}_{3}$ soundings compared with lidar measurements using the conservative coordinates reconstruction technique, Geophys. Res. Lett., 21, 1535-1538, 1994.

Redaelli, G.: Tecniche lagrangiane per l'analisi di misure in stratosfera, Ph.D. Thesis, pp. 105, University of L'Aquila, Italy, 1997.

Redaelli, G., Rizi, V., Molisani, P., and Visconti, G.: A box model simulating the evolution of polar stratospheric clouds and their chemical effects in the stratosphere. A preliminary test case study, in: Proc. of Quadrennial Ozone Symposium, L'Aquila, Italy, (Eds) Bojkov, R. and Visconti, G., 763-766, 1998.

Rodriguez, J. M., Ko, M. K. W., Sze, N. D., et al.: Nitrogen and chlorine species in the spring Antarctic stratosphere: comparison of models with airborne Antarctic ozone experiment observations, J. Geophys. Res., 94, 16683-16 703, 1989.

Rood, R. B., Nielsen, J. E., Stolarski, R. S., Douglass, A. R., Kaye, J. A., and Allen, D. J.: Episodic Total Ozone Minima and Associated Effects oh Heterogeneous Chemistry and Lower Stratospheric Transport, J. Geophys. Res., 97, 7979-7996, 1992.

Santee, M. L., Manney, G. L., Froidevaux, L., Zurek, R. W., and Waters, J. W.: MLS observations of $\mathrm{ClO}$ and $\mathrm{HNO}_{3}$ in the 1996 97 Arctic Polar Winter Geophys. Res. Lett., 24, 2713-2716, 1997.

Shindell, D. T., Ring, D., and Lonergan, P.: Increased polar stratospheric ozone losses and delayed eventual recovery owing to increasing greenhouse-gas concentrations, Nature, 392, 589-592, 1998.

Steil, B., Dameris, M., Bruhl, C., Crutzen, P. J., Grewe, V., Ponater, M., and Sausen, R.: Development of a chemistry module for GCMs: first results of a multiannual integration, Ann. Geophysicae, 16, 205-228, 1998.

Swinbank, R. and O'Neill, A.: A stratosphere-troposphere data assimilation system, Mon. Wheater Rev., 122, 686-702, 1994.

Van Leer, B.: Towards the ultimate conservative difference scheme. II: Monotonicity and conservation combined in a second-order scheme, J. Comput. Phys., 14, 361-370, 1974.

Visconti, G. and Pitari, G.: Seasonal and Latitudinal Distribution of Trace Gases in the Stratosphere: Results from a 2-D Residual Circulation Model, J. Atmos. Chem., 5, 255-289, 1987.

World Meteorological Organization (WMO): Rep. 25, World Meteorol. Org., Geneva, 1991. 